\title{
Modons on Tidally Synchronised Extrasolar Planets
}

\author{
J. W. Skinner, ${ }^{1 \star}$ J. Y-K. Cho, ${ }^{1,2,3} \dagger$ \\ ${ }^{1}$ School of Physics and Astronomy, Queen Mary University of London, Mile End Road, London E1 4NS, UK \\ 2 Department of Astrophysical Sciences, Princeton University, 4 Ivy Lane, Princeton, NJ, 08544,USA \\ ${ }^{3}$ CCA, Flatiron Institute, 162 Fifth Ave, New York, NY, 10010, USA §
}

Accepted yyyy mmm dd. Received yyyy mmm dd; in original form yyyy mmm dd

\begin{abstract}
We investigate modons on tidally synchronised extrasolar planets. Modons are highly dynamic, coherent flow structures composed of a pair of storms with opposite signs of vorticity. They are important because they divert flows on the large-scale; and, powered by the intense irradiation from the host star, they are planetary-scale sized and exhibit quasi-periodic lifecycles - chaotically moving around the planet, breaking and reforming many times over long durations (e.g. thousands of planet days). Additionally, modons transport and mix planetaryscale patches of hot and cold air around the planet, leading to high-amplitude and quasiperiodic signatures in the disc-averaged temperature flux. Hence, they induce variations of the "hot spot" longitude to either side of the planet's sub-stellar point - consistent with observations at different epoch. The variability behaviour in our simulations broadly underscores the importance of accurately capturing vortex dynamics in extrasolar planet atmosphere modelling, particularly in understanding current observations.
\end{abstract}

Key words: hydrodynamics - turbulence - methods: numerical - planets: atmospheres.

\section{INTRODUCTION}

Modons are translating vortex dipole structures, consisting of two oppositely-signed patches of vorticity that form in the atmosphere and ocean (Stern 1975). In atmospheric dynamics, modons play an important role in establishing "blocking patterns" which prevent weather or jet systems from moving through over a certain region. In a tidally synchronised planet atmosphere, a couplet of modons forms - one composed of a pair of cyclones and the other composed of a pair of anti-cyclones: cyclones (anti-cyclones) are storms characterised by vorticity having the same (opposite) sense as the planetary vorticity (e.g. Holton 2004). These modons are planetary-scale in size, and they initially span across the equator (assuming zero obliquity for the planet). While both modons are spatially compact and isolated, they generally interact strongly with each other as well as other flow structures present in the atmospheres of the synchronised planets because of the large interaction length-scale (the Rossby deformation scale $L_{\mathcal{R}}$ ) for the flow structures.

Currently, observations of hot-Jupiter atmospheres show large variations in the longitudinal location of the "hot spot" as well as the amplitude of spectral features (e.g. Grillmair et al. 2008; Zellem et al. 2014; Armstrong et al. 2017; Dang et al. 2018; Zhang et al. 2018; Jackson et al. 2019); see also Cho et al. (2019) for a recent review. However, atmospheric flow simulations that use the

\footnotetext{
* E-mail: j.w.skinner@qmul.ac.uk

† E-mail: jcho@flatironinstitute.org

$\ddagger$ on leave from Queen Mary University of London

$\S$ present address
}

commonly-employed forcing and initialization generally produce a large monolithic patch of nearly stationary hot area located eastward of the sub-stellar point $\sim 2 \times 10^{-3} \mathrm{MPa}$ (Cooper \& Showman 2005; Showman et al. 2008). The discrepancy between observations and simulations may arise because simulations thus far have lacked the required horizontal resolution (Skinner \& Cho 2021). Hence the simulations have not been able to accurately capture the dynamics of small-scales which influence the large-scale dynamics (e.g. modons and jets) through their non-linear interactions with the large-scales. Accurately modelling these interactions is essential because dynamics serves as the core for other important atmospheric processes such as radiative transfer, clouds, photochemistry, and ionization.

In this paper, we describe the results from a set of highresolution simulations, which accurately capture the small-scale vortices (storms) and waves inherent in synchronised planet atmospheres - hence, accurately represent large scale dynamics. Our results are grounded in a series of extensive numerical convergence and parameter sensitivity studies with the code used in the present work (Polichtchouk and Cho 2012; Polichtchouk et al. 2014; Cho, Polichtchouk \& Thrastarson 2015; Skinner \& Cho 2021). We find that tidally synchronised planet atmospheres contain a large number of intense storms, that span a wide range of sizes - including the planetary-scale. Significantly, these planetary-scale storms greatly influence the large-scale spatial distribution and temporal variability of hot, as well as cold, regions over the planet. These storms and their motions lead to signatures that may be observable and, therefore, important for interpreting and guiding current and future observations (Cho, Skinner \& Thrastarson 2021). 
The outline of this paper is as follows. In Section 2, we describe the numerical model we use, how it is set up for the simulations described in this work and linear theory relevant for the nonlinear solutions obtained with the model. In Section 3, we describe the transient and persistent solutions that arise when the aforementioned setup, which is commonly-employed in extrasolar planet studies, is used. We emphasize that the primary focus of this paper is on the flow and its associated temperature distributions: results from a comprehensive study of numerical convergence and accuracy (which includes the high resolution and dissipation order employed in this work) is reported in Skinner \& Cho (2021), and analysis incorporating radiative transfer, chemistry, and aerosols will be described elsewhere. In particular, here we focus on the quasiperiodic behaviour of modons and their effects on the flow and temperature fields. Significantly, this behaviour produces discernible signatures in disc-averaged temperature fluxes. In Section 4, we conclude by briefly summarising this work and discussing its implications for extrasolar planet circulation modelling and observations.

\section{METHODOLOGY}

\subsection{Governing Equations}

The governing equations, numerical model, and simulation setup used in this paper are the same as in Skinner \& Cho (2021) and Cho, Skinner \& Thrastarson (2021). We reproduce the key points of the equations and model here for the reader's convenience. As in many past works, we solve the traditional primitive equations (see e.g. Salby 1996) in $\boldsymbol{x}=(\lambda, \phi, p)$ coordinates, representing (longitude, latitude, pressure) in this work. The equations read:

$$
\begin{aligned}
\frac{\mathrm{D} \boldsymbol{v}}{\mathrm{D} t} & =-\nabla_{p} \Phi-\left(\frac{u}{R_{p}} \tan \phi+f\right) \boldsymbol{k} \times \boldsymbol{v}+\mathcal{D}_{\boldsymbol{v}} \\
\frac{\partial \Phi}{\partial p} & =-\frac{1}{\rho} \\
\frac{\partial \omega}{\partial p} & =-\nabla_{p} \cdot \boldsymbol{v} \\
\frac{\mathrm{D} T}{\mathrm{D} t} & =\frac{\omega}{\rho c_{p}}+\frac{\dot{q}_{\text {net }}}{c_{p}}+\mathcal{D}_{T}
\end{aligned}
$$

where $\mathrm{D} / \mathrm{D} t \equiv \partial / \partial t+\boldsymbol{v} \cdot \nabla_{p}+\omega \partial / \partial p$ is the material derivative; $t$ is the time; $\boldsymbol{v}=(u, v)$ is the (eastward, northward) velocity (in a frame rotating with rotation rate $\Omega$ ) on a constant $p$ surface; $\omega \equiv \mathrm{D} p / \mathrm{D} t$ is the "vertical" pressure velocity in the rotating frame; $R_{p}$ is the planetary radius, which is fiducially set to be at $p=0.1 \mathrm{MPa}$; $\boldsymbol{k}$ is the unit vector in the local vertical direction; $\nabla_{p}$ is the horizontal gradient on a constant $p$-surface; $\Phi(\boldsymbol{x}, t)=g z(\boldsymbol{x}, t)$ is the geopotential, where $g$ is the constant surface gravity at $z=R_{p}$ with $z$ the vertical distance above $R_{p}$; $f(\phi)=2 \Omega \sin \phi$ is the Coriolis parameter, the projection of the planetary vorticity vector $2 \Omega$ onto $k$; the direction of $\Omega$ orients north; $T(\boldsymbol{x}, t)$ is the temperature; $\mathcal{D}_{\chi}$, for $\chi \in\{\boldsymbol{v}, T\}^{1}$, is given by

$\mathcal{D}_{\chi}=\nu_{2 \mathfrak{p}}\left[(-1)^{\mathfrak{p}+1} \nabla_{p}^{2 \mathfrak{p}}+\mathcal{C}\right] \chi$,

where $\nu_{2 \mathfrak{p}}$ is the constant dissipation coefficient; $\mathfrak{p} \in \mathbb{N}$, where $\mathbb{N}=\{0,1,2, \ldots\}$, is the order of the dissipation (not to be confused with the pressure $p$ ) with $\mathfrak{p}>1$ instantiations known as

1 As in Skinner \& Cho (2021), $\{\cdot, \cdot, \ldots\},[\cdot, \cdot]$ and $(\cdot, \cdot, \ldots)$ carry their usual meanings in this paper - i.e. set, (closed) interval and tuple, respectively. hyper-dissipation (see e.g. Cho and Polvani 1996a; Thrastarson \& Cho 2011; Polichtchouk and Cho 2012); $\mathcal{C}=\left(2 / R_{p}^{2}\right)^{\mathfrak{p}}$ is a term that compensates the damping of uniform rotation by $\mathcal{D}_{v}$ (see e.g. Polichtchouk et al. 2014); $\rho(\boldsymbol{x}, t)$ is the density; $c_{p}$ is the constant specific heat at constant pressure; and, $\dot{q}_{\text {net }}(\boldsymbol{x}, t)$ is the net diabatic heating rate.

Equations (1) are closed by the equation of state for an ideal gas, $p=\rho \mathcal{R} T$, where $\mathcal{R}$ is the specific gas constant. A useful variable is the potential temperature, $\Theta(\boldsymbol{x}, t) \equiv T\left(P_{\text {ref }} / p\right)^{\kappa}$, where $p_{\text {ref }}$ is a constant reference pressure and $\kappa \equiv \mathcal{R} / c_{p}$; for example, $\Theta$ is materially conserved when $\dot{q}_{\text {net }}=\mathcal{D}_{T}=0$. The boundary condition for the equations is "free-slip" (i.e. $\mathrm{D} p / \mathrm{D} t=0$ ) at the top and bottom $p$-surfaces; note that the top and bottom boundaries are material surfaces, across which no mass is transported. With this boundary condition, the equations permit the full range of largescale motions for a stably-stratified, un-ionized atmosphere - with the exception of sound waves: sound waves are filtered out from the full compressible hydrodynamics equations ${ }^{2}$ via the combination of the hydrostatic balance condition, expressed by equation (1b), and the free-slip boundary conditions at the top and bottom. However, the results presented in this study (e.g. the emergence of dynamic modons) also apply to simulations solving the full NavierStokes (non-hydrostatic) equations employing a similar physical setup, since the speed of fast gravity waves admitted by both the hydrostatic and non-hydrostatic equations are close to the speed of the sound wave (see Table 1).

\subsection{Numerical Model}

We solve equations (1) and (2) numerically using the pseudospectral code, BOB (Rivier, Loft \& Polvani 2002; Scott et al. 2004). BOB is a highly-accurate "dynamical core" of a general circulation model (GCM). GCMs are typically used in atmospheric dynamics studies and climate modelling of the Solar System planets. But, BOB has been rigorously tested and validated under the numerically stringent conditions typical of hot-Jupiters; see, for example, Polichtchouk and Cho (2012), Polichtchouk et al. (2014), Cho, Polichtchouk \& Thrastarson (2015) and Skinner \& Cho (2021).

$\mathrm{BOB}$ solves the equations in the "vorticity-divergence and potential temperature" form ${ }^{3}$. In this form, equations (1) are more suited to the spectral transform method (see e.g. Orszag 1970; Eliasen et al. 1970; Canuto et al. 1988), which offers superior convergence properties compared to the traditional (e.g. finite difference) schemes (e.g. Boyd 2000; Durran 2010). BOB is essentially a multi-layer extension of the 1-layer codes used in the studies of Solar System giant planets by Cho and Polvani (1996a,b) and extrasolar system giant planets by Cho et al. (2003, 2008). The time integration of the equations in all of these codes is performed using a second-order accurate, leap-frog scheme with a small amount of Robert-Asselin filter applied to suppress the computational mode arising from the scheme (Robert 1966; Asselin 1972). The time-step size $\Delta t$ in all the simulations are such that the Courant-Friedrichs-Lewy (CFL) number (e.g. Strikwerda 2004; Durran 2010) is well below unity - typically $<0.3$.

For each $p$-surface, the code transforms the equations to the spectral space with a "triangular truncation" - i.e. up to $N=M \equiv$

\footnotetext{
2 Although sound waves are not admitted, the primitive equations are still compressible, as $\mathrm{D} \rho / \mathrm{D} t \neq 0$; see equation (1c).

3 the curl and divergence of equation (1a), along with equation (1d) in terms of the potential temperature
} 
Table 1. Physical, Numerical, and Scale Parameters: ${ }^{(a)}$ based on $c_{p}$; ${ }^{(b)}$ for $\left\{\mathrm{H}_{2}, \mathrm{He}\right\}$; ${ }^{(c)}$ at $p=0.1 \mathrm{MPa}$; ${ }^{(d)}$ at $p=1 \mathrm{KPa}$

\begin{tabular}{|c|c|c|c|}
\hline Planetary rotation rate & $\Omega$ & $2.1 \times 10^{-5}$ & $\mathrm{~s}^{-1}$ \\
\hline Planetary radius & $R_{p}$ & $10^{8}$ & $\mathrm{~m}$ \\
\hline Surface gravity & $g$ & 10 & $\mathrm{~ms}^{-2}$ \\
\hline Specific heat at constant $p$ & $c_{p}$ & $1.23 \times 10^{4}$ & $\mathrm{~J} \mathrm{~kg}^{-1} \mathrm{~K}^{-1}$ \\
\hline Specific gas constant ${ }^{(a, b)}$ & $\mathcal{R}$ & $3.5 \times 10^{3}$ & $\mathrm{~J} \mathrm{~kg}^{-1} \mathrm{~K}^{-1}$ \\
\hline Initial temperature $^{(c)}$ & $T_{m}$ & 1600 & $\mathrm{~K}$ \\
\hline "Equil." sub-stellar temp. ${ }^{(c)}$ & $T_{e_{\mathrm{d}}}$ & 1720 & K \\
\hline "Equil." anti-stellar temp. ${ }^{(c)}$ & $T_{e_{\mathrm{n}}}$ & 1480 & K \\
\hline Thermal relax. time ${ }^{(d)}$ & $\tau_{\mathrm{th}}$ & $\approx 10^{5}$ & $\mathrm{~s}$ \\
\hline Pressure at top & $p_{\text {top }}$ & 0 & MPa \\
\hline Pressure at bottom & $p_{\text {bot }}$ & {$[0.1,10]$} & $\mathrm{MPa}$ \\
\hline Pressure w/o forcing & $p_{0}$ & $\geqslant 1$ & $\mathrm{MPa}$ \\
\hline Truncation wavenumber & $\mathrm{T}$ & {$[21,682]$} & \\
\hline Number of levels (or layers) & $\mathrm{L}$ & {$[3,1000]$} & \\
\hline Max. sectoral wavenumber & $M$ & $=\mathrm{T}$ & \\
\hline Max. total wavenumber & $N$ & $=\mathrm{T}$ & \\
\hline Dissipation operator order & $\mathfrak{p}$ & {$[1,8]$} & \\
\hline Viscosity coefficient & $\nu_{2 \mathfrak{p}}$ & (see text) & $\mathrm{m}^{2 \mathfrak{p}} \mathrm{s}^{-1}$ \\
\hline (Hyper)dissip. wavenumber & $n_{d(2 \mathfrak{p})}$ & (see text) & \\
\hline Vertical length scale & $\mathcal{H}$ & $\sim \mathcal{R} T_{m} / g$ & $\mathrm{~m}$ \\
\hline Horizontal length scale & $\mathcal{L}$ & $\gtrsim R_{p} / 20$ & $\mathrm{~m}$ \\
\hline Maximum jet speed & $\mathcal{U}$ & $\gtrsim 2 \times 10^{3}$ & $\mathrm{~ms}^{-1}$ \\
\hline Sound speed $(c)$ & $c_{s}$ & $\approx 2.8 \times 10^{3}$ & $\mathrm{~ms}^{-1}$ \\
\hline Dissipation time-scale & $\tau_{d}$ & $\sim 2 \times 10^{5}$ & $\mathrm{~s}$ \\
\hline Brunt-Väisälä frequency & $\mathcal{N}$ & $\sim 2 \times 10^{-3}$ & $s^{-1}$ \\
\hline Rossby number & $R_{\mathrm{O}}$ & $\equiv \mathcal{U} /(\Omega \mathcal{L})$ & \\
\hline Froude number & $F_{\mathrm{r}}$ & $\equiv \mathcal{U} / \sqrt{g \mathcal{H}}$ & \\
\hline Rossby deformation scale & $\mathcal{L}_{\mathcal{R}}$ & $\equiv \sqrt{g \mathcal{H}} / \Omega$ & $\mathrm{m}$ \\
\hline
\end{tabular}

$\mathrm{T}$ wavenumbers retained in the Legendre expansion,

$\xi(\lambda, \mu, t)=\sum_{n=0}^{N} \sum_{m=-M}^{M} \xi_{n}^{m}(t) Y_{n}^{m}(\mu, \lambda), \quad|m| \leqslant n$,

where $\xi$ is an arbitrary scalar field; $\mu \equiv \sin \phi ; n \in \mathbb{N}$ and $m \in \mathbb{Z}$ are the total and sectoral wavenumbers, respectively; $(N, M) \in \mathbb{N}^{2}$; $Y_{n}^{m}(\lambda, \mu) \equiv P_{n}^{m}(\mu) e^{i m \lambda}$ are the spherical harmonic functions; and, $P_{n}^{m}$ are the associated Legendre functions. The set $\left\{Y_{n}^{m}\right\}$ are the eigenfunctions of the spherical Laplacian operator:

$\nabla^{2} Y_{n}^{m}=-\left[\frac{n(n+1)}{R_{p}^{2}}\right] Y_{n}^{m}$,

where

$\nabla^{2}=\frac{1}{R_{p}^{2}}\left\{\frac{\partial}{\partial \mu}\left[\left(1-\mu^{2}\right) \frac{\partial}{\partial \mu}\right]+\frac{1}{1-\mu^{2}} \frac{\partial^{2}}{\partial \lambda^{2}}\right\}$.

The $\left\{Y_{n}^{m}\right\}$ constitutes a complete, orthogonal expansion basis (e.g. Byron \& Fuller 1992). Note that, when $\mathfrak{p}=1$, equation (2) reduces to the Laplacian operator acting on $\chi$, modulo $\mathcal{C}$. Note also that a representation in spectral space with a truncation wavenumber $\mathrm{T}$ is transformed to a Gaussian grid in physical space with approximately $(3 \mathrm{~T}, 3 \mathrm{~T} / 2)$ points in the $(\lambda, \phi)$-direction. However, the Gaussian grid should not be directly compared with the grid of a finite-difference (or other grid-based) methods, as the former grid is effectively equivalent to a much higher resolution than a finitedifference grid with the same number of points. This is due to the pseudospectral method's accuracy and convergence properties and the use of $\mathfrak{p} \gg 1$ (e.g. Cho and Polvani 1996a; Boyd 2000; Durran 2010; Skinner \& Cho 2021).

Since our goal is to follow the evolution of highly dynamic and minimally dissipated flow structures over a long duration, we use $\mathfrak{p}=8$. The role of $\mathcal{D}_{\chi}$ is to limit dissipation to the small-scales and to provide a conduit for energy and enstrophy cascade that prevent the simulation from "blowing up". As has been demonstrated in Skinner \& Cho (2021), $\mathfrak{p} \geqslant 8$ is required at the currently practical resolutions to adequately capture the full range of behaviours exhibited in the flow. Using a lower $\mathfrak{p}$, especially at low resolution, can dissipate large-scale structures - even the $n=2$ mode structures, important in the present work. Other than the very weak Robert-Asselin filter to separate out the computational mode arising from the leapfrog scheme (Thrastarson \& Cho 2011), no other numerical dissipators, drags, fixers, stabilisers or filters are used in performing the simulations, as they are not necessary in our code.

Vertically, the domain is decomposed into $\mathrm{L} \in \mathbb{Z}^{+}$uniformlyspaced points or layers in the $p$-coordinate. Along this direction, a second-order finite-difference scheme is used - as is common in codes solving equations (1) (e.g. Durran 2010). Given the range, $p \in\left[p_{\text {top }}, p_{\text {bot }}\right]$, the dynamically active levels $p_{k}$ for $k \in[1, \mathrm{~L}]$ are located at

$p_{k}=\left(k-\frac{1}{2}\right)\left[\frac{p_{\text {bot }}-p_{\text {top }}}{\mathrm{L}}\right]$.

As already mentioned, the bounding surfaces, $p_{\text {top }}$ and $p_{\text {bot }}$, are not dynamically active; but, they enforce the boundary conditions. Note that many studies employ a $\log (p)$-spacing (e.g. Liu \& Showman 2013); but, they tend to solve the equations in a uniform $p$-coordinate, which introduces a numerical complication (Cho, Polichtchouk \& Thrastarson 2015). This difference in the vertical spacing, however, does not alter the main results of the present paper.

\subsection{Simulation Setup}

The physical parameters and their values for equation (1) are shown in Table (1). The parameter values are representative of the tidally synchronised extrasolar giant planet HD209458b. Note that the values in the table are identical to those used in many hot-Jupiter modelling studies (see e.g. Thrastarson \& Cho 2010; Skinner \& Cho 2021). This facilitates equatable comparisons. Note also that, due to the free-slip boundary condition at $p_{\text {bot }}$, the results in this paper are relevant to telluric planets with a solid or liquid surface, away from their boundary layers. ${ }^{4}$

For the thermal forcing of the planet's atmosphere, we adopt a commonly utilised scheme that has been implemented in many past studies (see e.g. Showman \& Guillot 2002; Cho et al. 2003; Cooper \& Showman 2005; Cho et al. 2008; Showman et al. 2008; Menou \& Rauscher 2009; Rauscher \& Menou 2010; Thrastarson \& Cho 2010; Heng et al. 2011; Liu \& Showman 2013; Cho, Polichtchouk \& Thrastarson 2015; Skinner \& Cho 2021). In this idealised setup, an atmosphere which is initially at rest is driven by a thermal relaxation to a prescribed "equilibrium temperature". Specifically, $\dot{q}_{\text {net }} / c_{p}$ in equation (1d) is set to be $-\left(T-T_{\text {eq }}\right) / \tau_{\text {th }}$, where $T_{\text {eq }}=T_{\text {eq }}(\lambda, \phi, p)$ is the equilibrium temperature and

4 Here, by 'telluric' we mean a planet with a solid lower boundary (and smaller radius). 
$\tau_{\mathrm{th}}=\tau_{\mathrm{th}}(p)$ is the radiative "cooling" time ${ }^{5}$; more precisely, it is the thermal relaxation time. The $T_{\text {eq }}$ distribution is specified as:

$T_{\mathrm{eq}}= \begin{cases}T_{e_{\mathrm{n}}}(p)+\Delta T(p) \cos \lambda \cos \phi, & \text { day-side } \\ T_{e_{\mathrm{n}}}(p), & \text { night-side } .\end{cases}$

Here $T_{e_{\mathrm{n}}}$ is the equilibrium temperature at the night-side, which is uniform at each $p$. In contrast, the day-side equilibrium temperature is not uniform, with $T_{e_{\mathrm{n}}}+\Delta T \equiv T_{e_{\mathrm{d}}}(p)$ the temperature at the sub-stellar point, $(\lambda, \phi)=\left(0^{\circ}, 0^{\circ}\right)$, for $\Delta T \equiv T_{e_{\mathrm{d}}}-T_{e_{\mathrm{n}}}$. The specified, simple profiles $\left\{T_{\text {ref }}, \Delta T, T_{e_{\mathrm{n}}}, T_{e_{\mathrm{d}}}, \tau_{\text {th }}\right\}$ all depend only on $p$ and crudely represent the effects of irradiation from the planet's host star on the dynamics. The profiles are shown in Fig. 1.

In Fig. 1, each of the profiles are piece-wise continuous functions of $p$ that span the vertical range, $p=\left[10^{-5}, 10^{1}\right] \mathrm{MPa}$. It is important to note that for this setup the strength of the thermal forcing is flow independent, which is not realistic (especially when $\tau_{\text {th }} \lesssim \tau_{\text {ad }}$, where $\tau_{\text {ad }} \equiv \mathcal{L} / \mathcal{U}$ with $\mathcal{L}=\pi R_{p}$ is the advective time). Moreover, the unknown $\tau_{\operatorname{th}}(p)$ is chosen so that the forcing strength is monotonic and strongest at the top of the atmosphere and goes to zero at $p=1 \mathrm{MPa}$; at $p \geqslant 1 \mathrm{MPa}$, forcing is not applied. Note that quantitative aspects of the flow are affected by the chosen profiles (as well as by the initial flow state) - as emphasized repeatedly in the past (Cho et al. 2008; Thrastarson \& Cho 2010; Cho, Polichtchouk \& Thrastarson 2015; Cho et al. 2019). Another feature to note is the zonal ${ }^{6}$ asymmetry of $T_{\text {eq }}$, which is responsible for generating the main (zonally-asymmetric) flow structure discussed in this paper: the cyclonic and anti-cyclonic modon pair. "Deeper asymmetry" about the terminator in $T_{\text {eq }}$, such as the $\cos \lambda$ distribution extending to the nightside (e.g. Thrastarson \& Cho 2010; Showman \& Polvani 2011), leads to stronger asymmetry in the flow. The effects of the asymmetry are discussed more in detail in section 3 .

Fig. 2 shows three useful profiles derived from the temperature profiles presented in Fig. 1: level mean atmospheric scale height, $H(p) \equiv\left(\mathcal{R} \int_{k}^{k+1} T \mathrm{~d} \ln p\right) /\left(g \int_{k}^{k+1} \mathrm{~d} \ln p\right)$, in units of Mm (left); potential temperature, $\Theta(p) \equiv T(10 / p)^{\kappa}$, in units of $\mathrm{K}$ (centre); and, Brunt-Väisälä frequency, $N(p) \equiv$ $\left[-\rho g^{2}(\mathrm{~d} \ln \Theta / \mathrm{d} p)\right]^{1 / 2}$, in units of $10^{-3} \mathrm{~s}^{-1}$ (right). The black lines correspond to the initial profile $T_{\text {ref }}$; and, the blue and red lines correspond to equilibrium profiles $T_{\mathrm{eq}}$ of the anti-stellar and sub-stellar points, respectively. The temperature profiles lead to abrupt changes in the vertical $\Theta$ gradients - i.e. jumps in the basic stratification - as seen in the Brunt-Väisälä frequencies. Note, the blue profile nearly vanishes at $p \approx 10^{-4} \mathrm{MPa}$, but it is still positive. It is also an artefact of the unrealistic discontinuity in the corresponding temperature profile. Formally, the hydrostatic balance condition restricts the validly of equations (1) to the stablystratified radiative region $p \lesssim 10 \mathrm{MPa}$, which overlies an unstablystratified convective region $p \gtrsim 10^{2} \mathrm{MPa}$. The exact transition level between the stable and unstable region is uncertain, and likely laterally dependent.

From hereon, the planetary radius $R_{p}$ and rotation period $\tau$ $\left(\equiv 2 \pi / \Omega=3.025 \times 10^{5} \mathrm{~s}\right)$ are used as the length and time scales, respectively - whenever confusion is not engendered. That is, $\nu_{2 p}$ is given in the units of $R_{p}^{2 \mathfrak{p}} \tau^{-1}$ and $n$ is given in the units of $R_{p}^{-1}$, by

5 The "spin up" time varies with depth and vertical resolution in all simulations, but it is around 10 planetary days for the $p_{\text {bot }} \sim 0.1$ simulations. For $p_{\text {bot }} \geqslant 1$, "spin up" time is undefined (Mendonça 2020).

6 "Zonal" refers to eastward and "meridional" refers to northward (and sometimes more loosely east-west and north-south, respectively).

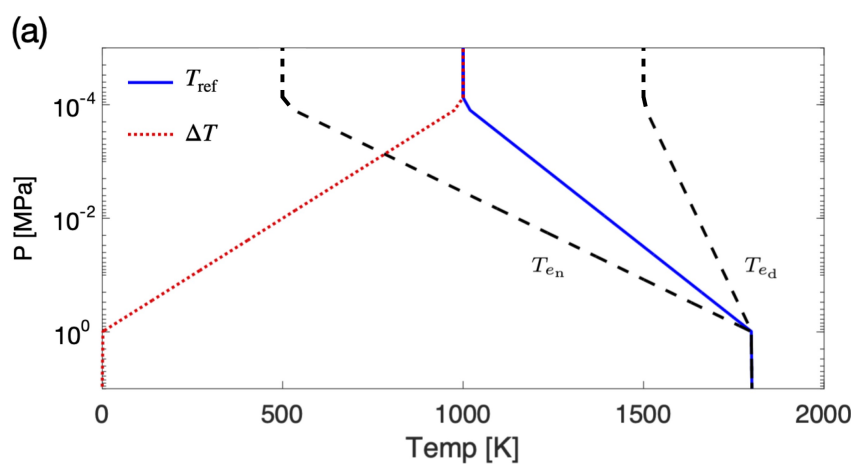

(b)

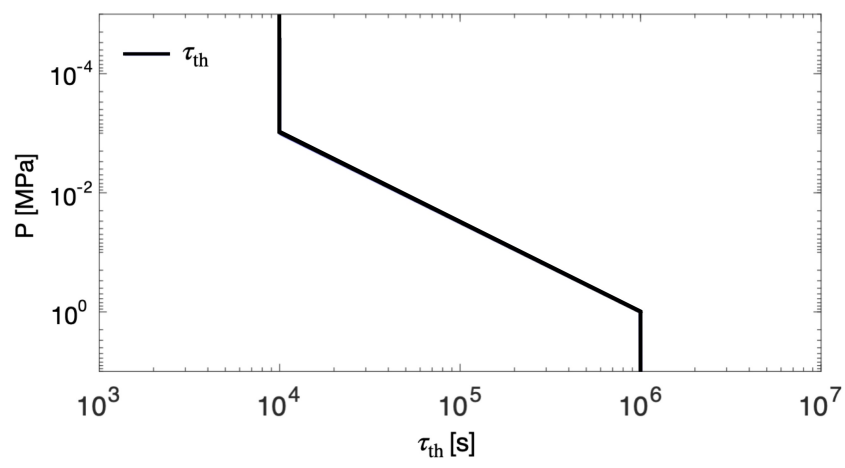

Figure 1. Temperature (a) and relaxation time (b) profiles used in the Newtonian cooling scheme. (a) Blue solid line shows the reference temperature $T_{\text {ref }}$, which is the initial temperature (uniform at each $p$ ). The accompanying black dashed lines show the equilibrium temperature profiles, $T_{e_{\mathrm{d}}}$ at the sub-stellar point (right) and $T_{e_{\mathrm{n}}}$ at the anti-stellar point (left); the latter temperature is a constant over the entire night-side at each $p$-level. The red dotted line is the equilibrium temperature difference, $\Delta T \equiv T_{e_{\mathrm{d}}}-T_{e_{\mathrm{n}}}$. (b) The thermal relaxation time $\tau_{\text {th }}$ is roughly proportional to $p / T_{\text {ref }}^{4}$ in the sloping region $\left(p=\left[10^{-3}, 1\right] \mathrm{MPa}\right)$ and uniform above and below $\left(\tau_{\mathrm{th}}\right.$ is a constant at each $p$-level). Outside the sloping region, $\tau_{\text {th }}$ is very short at the top ( $\tau_{\text {th }} \ll \tau_{\text {ad }}$ at $\left.p \leqslant 10^{-3} \mathrm{MPa}\right)$ and long at the bottom $\left(\tau_{\text {th }} \gg \tau_{\text {ad }}\right.$ at $p \geqslant 1 \mathrm{MPa}$ ).

default. However, the temperature $T$ and pressure $p$ are given the units of $\mathrm{K}$ and $\mathrm{MPa}$, respectively for easier comparison with past simulation and observatonal studies. Hence, for all the simulations discussed in this paper, $p_{\text {top }}=0$ while $p_{\text {bot }} \in\{0.1,1.0,10\}$. Note that a range of simulation domains are chosen to explore the robust features, for the given setup. Appropriate $p$-levels for the top and bottom of the simulations are currently unknown (Cho et al. 2008).

\subsection{Linear Theory}

Before embarking on the full non-linear solutions, a brief discussion of linear theory is instructive. Under the barotropic (vertically aligned) and isochoric (constant density) assumptions, equation (1) reduces to the shallow-water equations for a single layer of fluid (Pedlosky 1987). Here we confine our attention to a tangent plane situated at the equator $(\phi=0)$, known as the equatorial $\beta$-plane approximation (e.g. Matsuno 1966; Gill 1980; Wu, Sarachik \& Battisti 2001):

$$
\begin{aligned}
& \frac{\mathrm{D} \boldsymbol{v}}{\mathrm{D} t}=-g \nabla h-\beta y \boldsymbol{k} \times \boldsymbol{v}, \\
& \frac{\mathrm{D} h}{\mathrm{D} t}=-h \nabla \cdot \boldsymbol{v},
\end{aligned}
$$



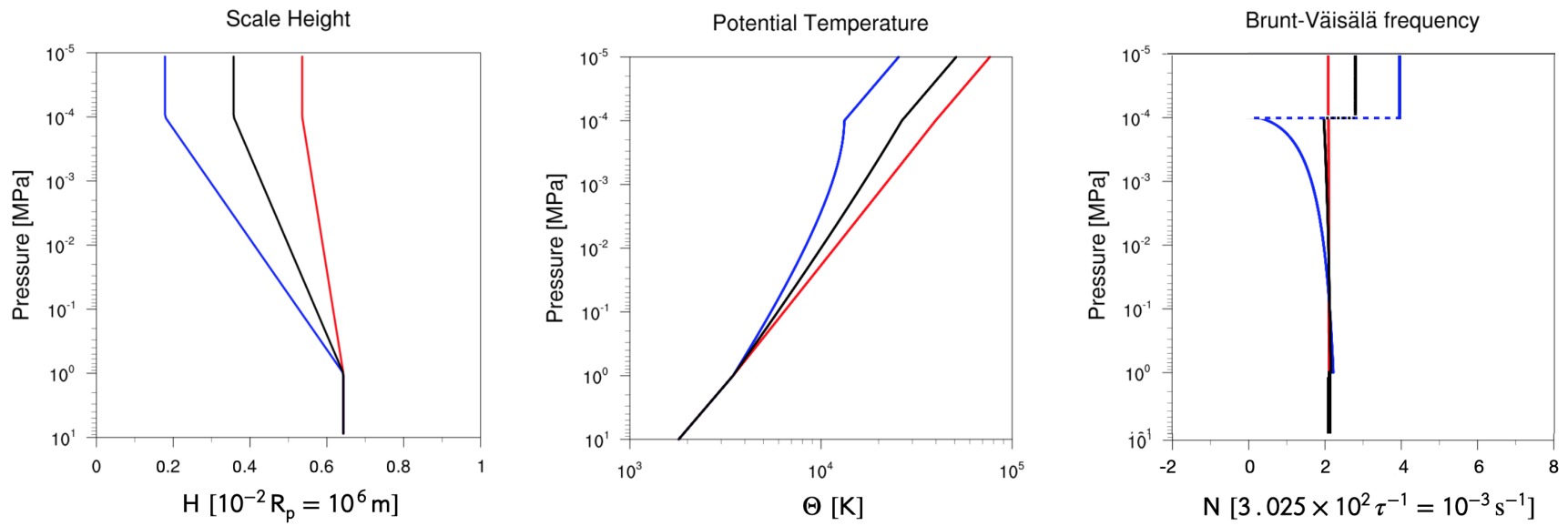

Figure 2. Level mean scale height $H(p)$ in units of $\mathrm{Mm}$ (left), potential temperature $\Theta(p)$ in units of K (centre), and Brunt-Väisälä frequency $N(p)$ in units of $10^{-3} \mathrm{~s}^{-1}$ (right) for the temperature profiles in Fig. 1. The black lines correspond to the initial profile $T_{\text {ref }}$; and, the blue and red lines correspond to equilibrium profiles of the anti-stellar and sub-stellar points $\left(T_{e_{\mathrm{n}}}\right.$ and $\left.T_{e_{\mathrm{d}}}\right)$, respectively. The temperature profiles lead to abrupt changes in the vertical $\Theta$ gradients - i.e. jumps in the basic stratification - as seen in the Brunt-Väisälä frequencies. In the latter, the blue profile nearly vanishes at $p \approx 10^{-4} \mathrm{MPa}$, but it is still positive.

where now $\mathrm{D} / \mathrm{D} t \equiv \partial / \partial t+\boldsymbol{v} \cdot \nabla$ with $\nabla \equiv(\partial / \partial x, \partial / \partial y)$; $\boldsymbol{v}=(u, v) \in \mathbb{R}^{2}$, where $u(\boldsymbol{x}, t)$ is the zonal velocity and $v(\boldsymbol{x}, t)$ is the meridional velocity; $h(\boldsymbol{x}, t)$ is the fluid thickness; $\beta \equiv$ $\mathrm{d} f /\left.\mathrm{d} y\right|_{y=0}=2 \Omega / R_{p}$ is the meridional gradient of the Coriolis parameter at the equator; and, $\boldsymbol{k}$ is again the unit vector in the vertical direction. With the boundary condition, $y \rightarrow \pm \infty$, the dynamics is effectively confined in the equatorial region because of the finiteness of $L_{\mathcal{R}}$; hence, the equatorial beta plane acts like a wave guide along the equator, with a number of different zonally propagating waves that are confined in the meridional direction and divided into fast and slow types. Importantly, equatorial waves in equations (1) have the same horizontal structure as those admitted by equations (8). However, we stress that the use of $\beta$-plane model here is used to motivate the explanation for the initial formation of the modon (only). The Matsuno-Gill solution for the equatorial $\beta$-plane (e.g. Showman \& Polvani 2011), for example, does not formally and realistically apply to hot synchronised planet atmospheres ${ }^{7}$ because of the following (Cho et al. 2019): $i$ ) large meridional scale of the modons invalidates the tangent plane approximation; ii) a pre-existing, strongly time-varying background flow and temperature field due to dynamic modons and other storms, which preclude classical eddy-mean flow interaction theory (e.g. Vallis 2017); iii) incompressible (small Mach number) and homogeneouslayer (unconstrained $L_{\mathcal{R}}$ ) assumptions of the shallow-water model; and, $i v$ ) hot-Jupiters are not expected to have a linear Rayleigh drag.

Upon non-dimensionalising equations (8) with the scaling, $(\boldsymbol{v}, \boldsymbol{x}, t) \rightarrow\left(U \boldsymbol{v}, L \boldsymbol{x}, \tau_{s} t\right)$, where $U, L$, and $\tau_{s} \equiv L / U$ are the characteristic speed, length, and time scales, respectively, and then linearising the equations to zeroth-order in the Rossby number,

7 particularly to explain the supersonic, zonally-symmetric, equatorial jet which develop after a long time in low-resolution simulations
$R_{o} \equiv U /\left(\beta L^{2}\right)$, we obtain:

$$
\begin{aligned}
& \frac{\partial u}{\partial t}-v y+\frac{\partial h}{\partial x}=0, \\
& \frac{\partial v}{\partial t}+u y+\frac{\partial h}{\partial y}=0, \\
& \frac{\partial h}{\partial t}+\frac{\partial u}{\partial x}+\frac{\partial v}{\partial y}=0 .
\end{aligned}
$$

From this, we can find a general solution for linear modes at the equator, by eliminating $u$ and $h$ and obtaining an equation for $v$ :

$\frac{\partial}{\partial t}\left(\nabla^{2} v-y^{2} v-\frac{\partial^{2} v}{\partial t^{2}}\right)+\frac{\partial v}{\partial x}=0$.

Now, expanding $v$ in a series in the Hermite polynomial basis $\varphi_{l}$ (Abramowitz \& Stegun 1965), $v=\sum_{l} v_{l}(x, t) \varphi_{l}(y)$, and using

$\frac{\mathrm{d}^{2} \varphi_{l}}{\mathrm{~d} y^{2}}+\left(2 l-y^{2}+1\right) \varphi_{l}=0$

gives the following equation:

$\frac{\partial}{\partial t}\left[\frac{\partial^{2} v_{l}}{\partial x^{2}}-(2 l+1) v_{l}-\frac{\partial^{2} v_{l}}{\partial t^{2}}\right]+\frac{\partial v_{l}}{\partial x}=0$

This leads to

$\frac{\partial^{3} \hat{v}_{l}}{\partial t^{3}}+\left(k^{2}+2 l+1\right) \frac{\partial \hat{v}_{l}}{\partial t}-i k \hat{v}_{l}=0$,

where $\hat{v}_{l}(k, t)=\int v_{l}(x, t) e^{i k x} \mathrm{~d} x+c . c$. is the Fourier transform of $v_{l}$. The general solution for equation (13) is:

$\hat{v}_{l}=\sum_{\alpha=1}^{3} v_{\alpha}(k) e^{-i \omega_{\alpha} t}$,

where $\omega_{\alpha}$ for $\alpha=1,2,3$ are the three roots of the dispersion equation:

$\omega_{\alpha}^{3}-\left(k^{2}+2 l+1\right) \omega_{\alpha}-k=0$.

Here, the lowest value of $\omega_{\alpha}$ at a given $(k, l)$ corresponds to a wave with westward propagation. This is the equatorial Rossby wave, important for the large-scale structure in our work. Note that $l$ gives 


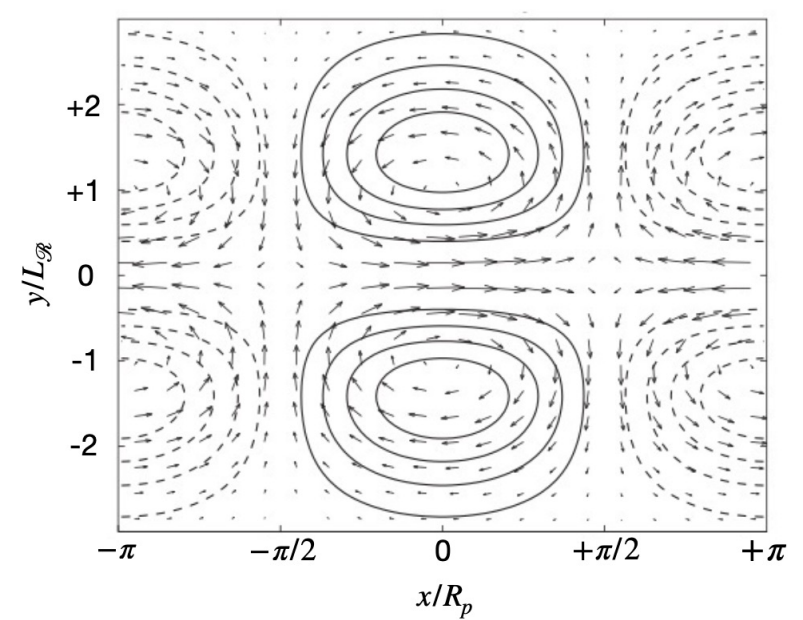

Figure 3. Velocity (vectors) and height perturbation (contours) distributions for the equatorial Rossby wave with $(k, l)=(1,1)$, illustrating the horizontal structure of the dominant flow pattern in this paper (e.g. bottom $p$-level of Fig. 4). Note the different scalings in the $x$ and $y$ directions, appropriate for the dynamics under discussion. For the contours, negative (positive) values are full (dashed); under geostrophy, height and vorticity perturbation fields are locally (and at the equator) related by the Laplacian and opposite signs in spectral amplitude.

the number of nodes in the meridional direction for $v$. The approximate solution to equation (15) is

$\omega_{l}(k)=\frac{k}{2 l+k^{2}+1}$.

The velocity and height perturbation distributions for the equatorial Rossby wave with $(k, l)=(1,1)$ from equation $(15)$ are shown in Fig. 3. The figure illustrates the horizontal structure of the dominant flow pattern in this paper, a modon pair. We note that Rossby waves generally have much lower phase velocities than inertia-gravity waves. In addition, in the long-wave part of the wave spectrum, there is a well-pronounced gap between inertia-gravity waves and the rest of the spectrum. In the next section, we show how such a structure is modified in its morphology and dynamical behaviour under full non-linearity and forcing at high resolution.

\section{RESULTS}

\subsection{Initial Structures}

Fig. 4 presents surfaces of relative vorticity $\left(\zeta= \pm 3\right.$, in units of $\tau^{-1}$ and at $\tau^{-1}$ intervals), centred at the sub-stellar point $(\lambda=0, \phi=0)$, at $t=0.25$. The red and blue surfaces correspond to positive- and negative-valued surfaces, respectively. The overall structure seen in the figure is a pair of vortex columns, each with opposite signs of $\zeta$ in the vertical direction. The vortex columns form almost immediately after the start of the simulations and then quickly (within a few $\tau$ 's) evolve to a simpler structure, as described below. In oceanography, such columnar structures are known as "hetons" (Hogg \& Stommel 1985). Hetons are generated as a direct response to the applied thermal forcing and potential vorticity (e.g. Pedlosky 1987) conservation, which is valid for this setup at early times and wherever the flow is in equilibration with the applied thermal forcing. The snapshot shown is from a T341L20 resolution simulation with a vertical domain range of $p \in[0,0.1]$ and uniformly-spaced layers of $\Delta p=5 \times 10^{-3}$ thickness.

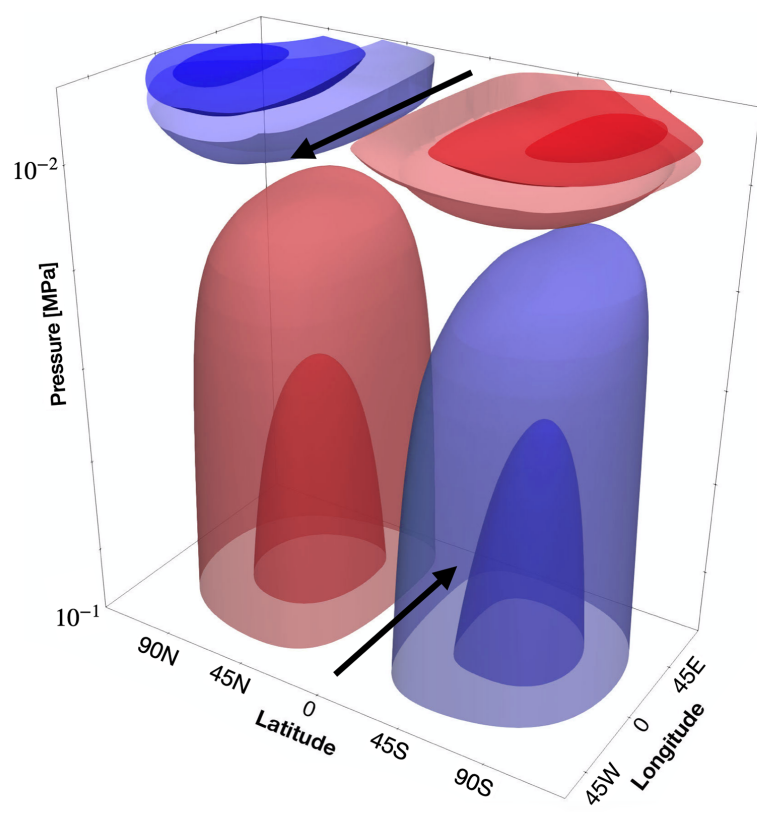

Figure 4. Relative vorticity surfaces $\zeta(\lambda, \phi, p)= \pm 3$ (in units of $\tau^{-1}$ and at $\tau^{-1}$ intervals, with positive values in red and negative values in blue), at $t=0.25$ (in units of $\tau$ ) from a T341L20 simulation: the dissipation order is $\mathfrak{p}=8$, viscosity coefficient is $\nu_{16}=1.5 \times 10^{-43}$ (in units of $R_{p}^{2 \mathfrak{p}} \tau^{-1}$ ), and time-step size is $\Delta t=4 \times 10^{-5}$ (in units of $\tau$ ). Black arrows show bulk velocity $(\sim 500 \mathrm{~m} / \mathrm{s})$. The section of the atmosphere shown is centred on the sub-stellar point $(\lambda, \phi)=(0,0)$ with vertical range $p \in[0,0.1]$ (in units of MPa). When the simulations are initialised as described in Section 2.3 (i.e. baroclinic thermal forcing applied to atmosphere at rest), a "hetonic quartet" forms at the sub-stellar point; a second quartet forms at the nightside. This baroclinic structure cannot be captured by a barotropic model (e.g. shallow-water model in Section 2.4). The quartet is composed of two oppositely-signed hetons, which here is anti-symmetric across the equator and separated in latitude by $\phi \sim 80^{\circ}$. Each heton is a columnar structure composed of a pair of similar strength and oppositely-signed vortices at different $p$ levels (which here are tilted in longitude by $\lambda \sim 20^{\circ}$ ). The heton quartet is a transient feature of the employed setup and is broken up by a strong vertical shear with the upper part of the atmosphere accelerating to a fast equatorial jet. The formation is independent of the location of the $p_{\text {bot }}$ considered - i.e. $p_{\text {bot }} \in[0.1,10]$.

As seen in Fig. 4, this early time flow structure is composed of a pair of hetons - i.e. a "hetonic quartet" (Kizner 2006). This hetonic quartet is a vortical quadrupole which forms in response to the specified thermal forcing. The two hetons, as a pair, straddle the equator and their centres are separated by a latitudinal distance of $\sim 80^{\circ}$. Both hetons are slightly tilted in the longitudinal direction, with the top vortical structure offset longitudinally by $\sim 20^{\circ}$ from the bottom vortical structure. Also, both hetons are cyclonic ${ }^{8}$ in the $p \lesssim 2.5 \times 10^{-2}$ region and anti-cyclonic in the $p \gtrsim 2.5 \times 10^{-2}$ region. Note that the $p$-level where the vorticity inversion occurs depends on the extent of the simulation's vertical domain (e.g. at a deeper level for larger domain range). Note also that, in addition to the heton quartet shown, a weaker and oppositely-signed heton quartet forms near the anti-stellar point; hence, there are actually two (anti-symmetric) hetonic quartets - i.e. a "hetonic octet". The

8 Cyclonicity is defined according to the sign of $\zeta \cdot \Omega$ : the sign is positive for cyclones and negative for anti-cyclones. 
night-side hetons are larger in areal extent laterally; this is due to the specified thermal forcing, which consists of relaxing the nightside temperature field to a uniform distribution that is much cooler than the day-side. Note that the octet formation is independent of the domain $p$-range considered in this work, from $\left[0,3 \times 10^{-2}\right]$ to $[0,20]$.

Overall, both quartets are strongly barotropic but quickly become baroclinic (vertically tilted) as the thermal forcing accelerates the flow at the top of the domain. As early as $t=1$, the nightside quartet migrates to the day-side and the two quartets interact strongly with each other at the eastern terminator. This results in intense baroclinic fronts that sweep across the eastern to western terminators, from the low latitude to the pole, and in both the northern and southern hemispheres (the fronts, at a later time, can be seen in Fig. 5). Significantly, the fronts undergo shear instability and act as sources of small-scale vortices (storms): wind speeds parallel to the fronts are already high, averaging $\sim 4.5$ (i.e. $\sim 1500 \mathrm{~m} \mathrm{~s}^{-1}$ ) at $p<5 \times 10^{-2}$. In general, sharp fronts play a seminal role in hot exoplanet atmospheric dynamics. For example, capturing the front at the early time (or any other time later) leads to a subsequent evolution which is markedly different than when not captured. The initially strong baroclinicity, eventually shears off the top of the heton until like-signed vortical structures vertically align; this leads to a strongly barotropic structure overall, dominated by the modons which grew in strength upward from the bottom. Thus, hetons are transient structures: they may also form at later times but are weaker and more short-lived, due to the ambient flow conditions (which is not at rest).

\subsection{Modon Pairs and Other Storms}

Fig. 5 presents the main result of this paper: given the idealised setup of a tidally synchronised exoplanet as described above, a pair of planetary-scale, strongly-barotropic modons is a generic solution to equation (1). Under the applied forcing, a pair of modons (cyclonic and anti-cyclonic) forms near the planet's sub-stellar point $(\lambda=0, \phi=0)$ and the anti-stellar point $(\lambda=180, \phi=0)$. Both modons straddle the equator, but the night-side modon (anticyclonic) is always weaker than the day-side modon (cyclonic), when they first form. The cyclonic modon can be clearly seen in the figure, which shows the $\zeta$-field at $t=100-$ well after the initial transient behaviour (generally lasting less than $\sim 10$ planetary days). The resolution of the simulation in the figure is T682L20; the dissipation order is $\mathfrak{p}=8$; the viscosity coefficient is $\nu_{16}=$ $2.3 \times 10^{-48}$; the time-step size is $\Delta t=2 \times 10^{-5}$; and, the extent of the vertical domain is $p \in[0,0.1]$. The two levels presented, which are from near the top and bottom of the simulation domain, show the strongly barotropic structure of the modon. Note that there is no $\zeta$ inversion between the $p$ levels shown in Fig. 5. We have performed an extensive convergence assessment with the present setup and have verified that the features and behaviours presented in this paper are qualitatively robust at, or above, T341 horizontal resolution with $\nabla^{16}$ hyper-viscosity (Skinner \& Cho 2021).

As can be seen in Fig. 5, the equilibrated flow for this setup is broadly characterised by three features: 1) a fast, prograde (eastward flowing), zonally asymmetric equatorial jet; 2) sharp, planetary-scale fronts which form in both the northern and southern hemispheres and roll up into small-scale vortices near the eastern terminator, particularly at the lower $p$-level; and, 3) planetary-scale storms that exhibit a variety of quasi-periodic stable states, as well as transitions between those states. We stress that, when the flow is adequately resolved, the equatorial jet is not zonally symmet- ric; this is in contrast with nearly all simulations in the past employing the same setup with lower resolution and/or viscosity order (e.g. Liu \& Showman 2013). Likewise for the unstable fronts; highresolution is required to capture this important source of mediumscale storms (Cho et al. 2003; Cho, Skinner \& Thrastarson 2021; Skinner \& Cho 2021). As for the planetary-scale storms, the most generic state is a vortical quadrupole (at a given $p$-level), of which at least one is a coherent modon (Fig. 5, and see also Figs. 6 and 8). In general, there are two coherent modons, one composed of a pair of cyclones and the other composed of a weaker pair of anticyclones, as already mentioned. In the figure, the storms comprising the cyclonic modon are separated meridionally by a centre-tocentre distance of $\sim \pi R_{p}$ : the storms of the anticyclonic modon are much less conjoined and located closer to the poles. Additionally, the cyclonic modon is generally shrouded by a sharp front to its east and a trailing Rossby wave mostly to its west (seen as undulations along fronts propogating westward from the cyclonic modon in Fig. 5b), both of which generate copious small-scale storms and gravity waves. This is part of the "geostrophic adjustment" process of a modon (e.g. Lahaye \& Zeitlin 2012). The multiple flow states will be described separately in more detail later.

Broadly, the flow features described above are seen at essentially all the $p$-levels. Unlike the transient hetons described above, neither of the cyclonic or anticyclonic modons in the figure changes its cyclonicity throughout the simulation. Modons are generally present near the top of the modelled atmosphere (Fig. 5a), although it is less prominent than at higher $p$-level (Fig. 5b): this is because of the stronger anisotropic turbulence at the top. Nevertheless, jets and fronts are both greatly influenced by the modons, independent of $p$. In particular, when the cyclonic modon is positioned near the sub-stellar point the equatorial jet generally steepens, undulates and then breaks on a time-scale of $\sim 3$ planetary days. This strongly non-linear behaviour is caused by the oscillation and rotation of the storms (that comprise the cyclonic modon) about their equilibrium positions. Note also how the two constituent storms have entrained small-scale storms from the breaking jet into their cores (e.g. Fig 5a), thus mixing low-latitude air (and temperature) into the mid-latitudes.

Each modon forms out of an initial hetonic quartet, which strengthens upward in time from the lower vortex after the tops of the original hetons are sheared away (as described in Section 3.1). The first separation can occur as early as $t=5$, resulting in chaotic mixing in the top region of the modelled atmosphere very quickly. Despite the meridional symmetry imposed by the initial and forcing conditions, the flow and mixing break this symmetry early on. The zonal symmetry is also broken early on, even before the zonally asymmetric forcing builds up its strength (recall the short relaxation time $\tau_{\text {th }}$ ). Both the modon and the front radiate large-amplitude gravity waves as well as induce formation of small-scale storms. The anti-cyclonic modons, which are less visible in Fig. 5, also radiate and generate small-scale structures, but these structures are weaker and more diffused.

After the initial formation, the modons execute a complex set of motions as they evolve non-linearly. The motions, however, exhibit distinct life-cycles, which may be observable. One of the cycles is associated with generation then decimation of modons repeating many times over 1500 planetary days of T170 simulations. ${ }^{9}$ Qualitatively same behaviour occurs in T341 and T682 resolution simulation over shorter durations (300 planetary days). Sig-

9 Given the lack of realistic physical parameterizations, such a long du- 


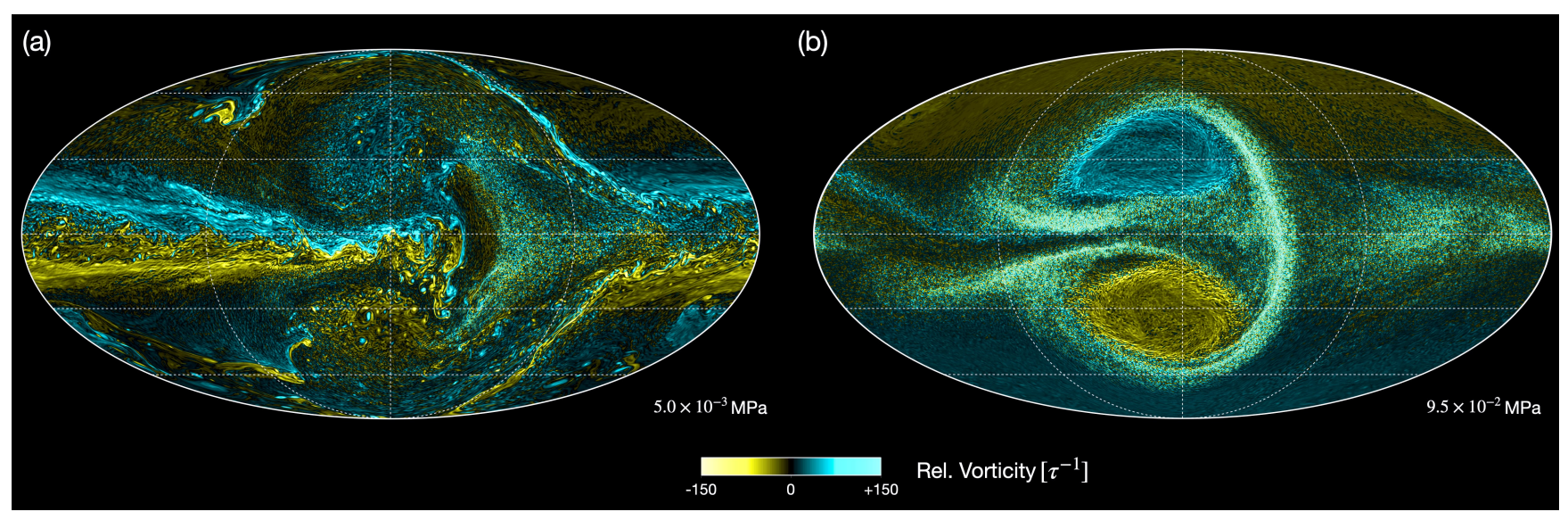

Figure 5. The relative vorticity $(\zeta)$ field from a T682L20 resolution simulation at $t=100$ in Mollweide projection. The dissipation order, viscosity coefficient and time-step size are $\mathfrak{p}=8, \nu_{16}=2.3 \times 10^{-48}$ and $\Delta t=2 \times 10^{-5}$, respectively. The $p$-levels shown, $5.0 \times 10^{-3}$ (a) and $9.5 \times 10^{-2}$ (b), correspond to the mid-points of the top and bottom layers of the computational domain, respectively. The flow is dominated by two highly dynamic, planetary-scale modons - a cyclonic modon at the sub-stellar point (at centres of the frames) and a much weaker and larger anti-cyclonic modon at the night side (the sides of the frames). The cyclonic modon straddles an undulating, zonally-asymmetric equatorial jet at both $p$-levels. The equatorial jet in a) is halted just to the east of the sub-stellar point and breaking throughout its "core" (along the equator): the jet core is where there is a jump in $\zeta$ in the meridional direction (near the equator). The equatorial jet in b), in contrast, is rolling up much more prominently at its northern and southern edges. The cyclonic modons in both frames emit large-amplitude gravity waves and generate thousands of small-scale vortices at their peripheries; the anticyclonic modons are barely visible at both $p$-levels because they are more diffused and lack sharp bounding fronts in these frames. Both cyclonic and anticyclonic modons are highly dynamic and exhibit periodic life-cycles in which they generally (but not always) migrate westward around the planet, while strongly interacting with other flow structures - e.g. storms, jets and waves.

nificantly, both cyclonic and anti-cyclonic modons are highly dynamic and chaotically translate westward, in general, while undergoing frequent changes in size, shape, orientation and strength as they do so. These behaviours can easily be missed when averaged fields or quantities are strictly used to study the atmosphere; see e.g. discussions in Cho, Polichtchouk \& Thrastarson (2015) and Cho et al. (2019). As already discussed, both hetons and modons can form in simulations encompassing a wide range of pressure levels. This includes simulations which include the deeper region, where the thermal forcing is not applied. This is shown explicitly in Fig. 6.

In Fig. 6, two "deep atmosphere" simulations (i.e. those with $\left.p_{\text {bot }} \geqslant 1\right)$ are presented. The resolution of the simulations is T170L200; and, $\mathfrak{p}=8, \nu_{16}=10^{-38}$ and $\Delta t=8 \times 10^{-5}$. The $p_{\text {bot }}$ is different between the two simulations, with values of 1 and 10 (left and right columns, respectively). The $\zeta$-field is displayed in all the frames. Fig. 6 a shows the top of a hetonic quartet, which forms in the deep atmosphere simulations at early times - as in the "shallow atmosphere" simulation (i.e. with $p_{\text {bot }}=0.1$, presented in Fig. 4). The meridional symmetry is strong at the time shown, but quickly breaks shortly thereafter. Figs. 6(b-d) present modons in deep atmosphere simulations at different $p$-levels and times. As can be seen, modons occur near the top of the modelled domain independently of the location of the domain bottom, provided a sufficient number of vertical levels are used to span the domain. The modons shown here also translate around the planet and periodically break and reform into several different configurations of vortices, similar to the behaviour in the shallow atmosphere simulations.

We note here that several past studies employing a similar setup have captured vortex dipole structures (e.g. Thrastarson \& Cho 2010; Heng et al. 2011; Cho, Polichtchouk \& Thrastarson

ration simulation should not be taken too literally - particularly given the short $\tau_{\mathrm{th}}$ in the upper part of the domain.
2015; Skinner \& Cho 2021). However modons were not the focus of these studies. Moreover, past studies were performed with lower resolution and/or stronger numerical viscosity than in the present study. Since capturing the fine-scale structures (and, in particular, their influence on the large-scale structures) is crucial, highresolution and minimal over-dissipation are necessary ingredients in accurately modelling modons. Specifically, having extensively investigated the influence of well-resolved fine-scale structures, we find the longevity, dynamism and multiple state behaviours of planetary-scale modons are fundamentally affected. Below the T170 horizontal resolution and when low-order viscosity and/or large viscosity coefficient are used, modons are markedly diffused and translate smoothly around the planet - if they move at all. Strict numerical convergence does not appear to be achieved until at least T341 resolution (Skinner \& Cho 2021).

Fig. 7 presents the longitude positions of the modon centroids from the T682L20 simulation in Fig. 5, as an illustrative example. Such plots are instructive, particularly for observations, because of the modons' strong influence in redistributing large patches of hot and cold air across the planet - as discussed more in detail below. In the figure, blue and red lines show the longitudinal distance traversed by the cyclonic and anticyclonic modons, respectively. Dotted lines indicate linear fits, with the $2 \pi$-traversal periods indicated in the legend. As already noted, the behaviours are quantitatively different at different resolution and dissipation order. However, the general behaviour here is very roughly similar down to T85 resolution - provided $\mathfrak{p}=8$ dissipation order is employed (Skinner \& Cho 2021): in particular, the cyclonic modon's westward translation is observed, but the modon is sluggish and is devoid of the non-linear or oscillatory motions (which arise when small-scale eddies are captured at T341 and above resolutions). Essentially all previous extrasolar planet simulations have been performed with less than T341 resolution and $\mathfrak{p}=8$ dissipation order - especially ones that use the current setup and are three dimensional. 


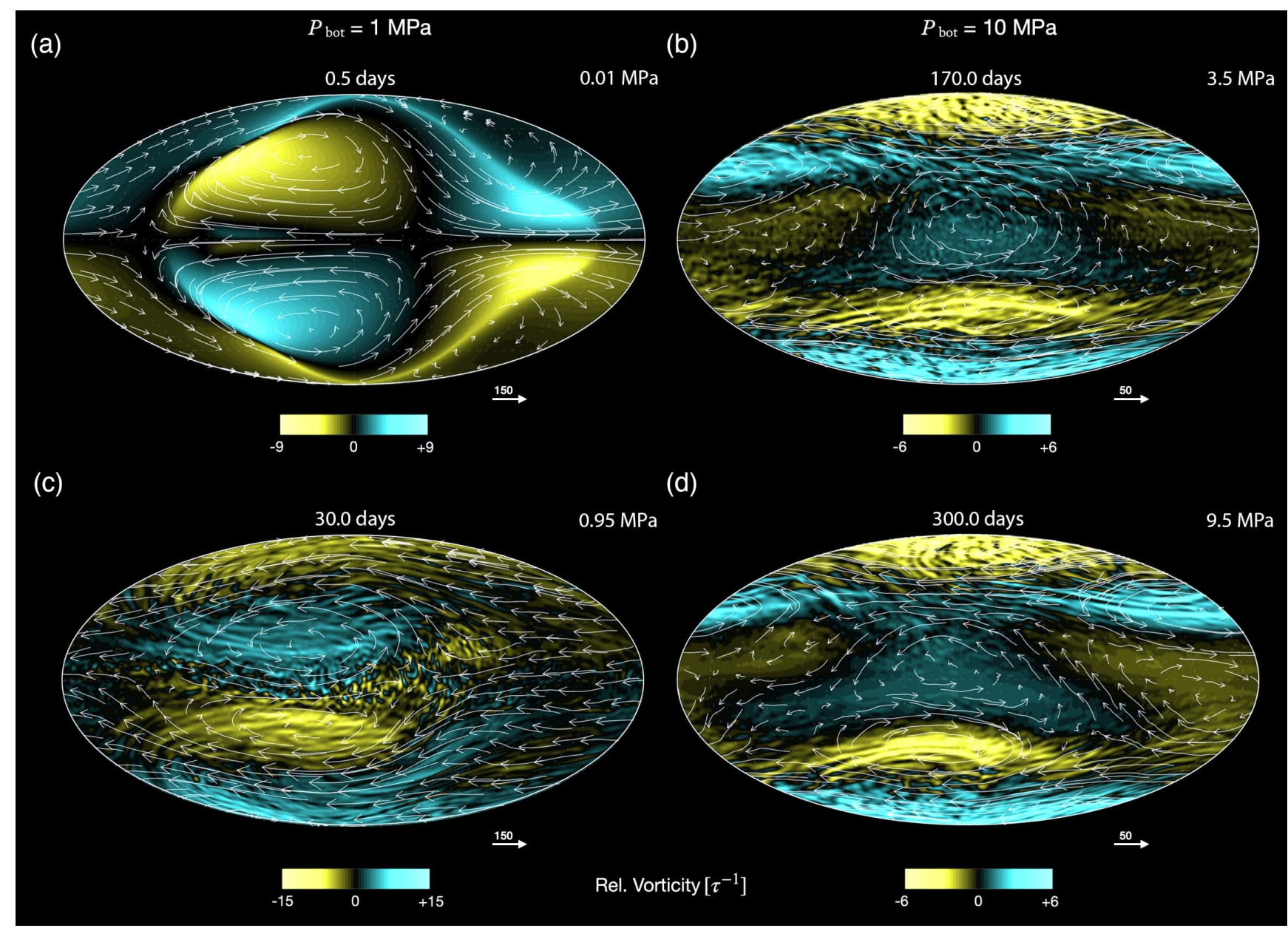

Figure 6. Two "deep atmosphere" simulations with $p_{\text {bot }}=1$ (left) and $p_{\text {bot }}=10$ (right) at different times (labeled, top). The resolution of both simulations are T170L200; both are set up identically and carried out with $\mathfrak{p}=8, \nu_{16}=10^{-38}$ and $\Delta t=8 \times 10^{-5}$. The $\zeta$ fields at the times indicated are shown in Mollweide projection centred on the sub-stellar point, and the times are chosen when the modons are clearly in view (near the sub-stellar point); the winds are overlaid with the reference vector length (in units of $\mathrm{m} \mathrm{s}^{-1}$, which is equal to $3.025 \times 10^{-3}$ in $R_{p} \tau^{-1}$ units), adjusted in each frame for visual clarity. Frame (a) shows the top of a hetonic quartet, which also forms in these deep simulations at early times (as in the "shallow atmosphere" simulations); the flow field is not yet turbulent at this time, but sharp fronts have already developed. Frames (b-d) show coherent modons are present at various times and at various $p$-levels. Post the initial transient stage, the modons evolve, embedded in turbulent fields containing many smaller scale flow structures - including gravity waves (striations in $\zeta$ ). Here modons generally migrate westward (chaotically) around the planet - periodically breaking or transitioning to different configuration states (again, as in the shallow atmosphere simulations). Broadly, the flow tends to be more zonal and often contains an extra planetary-scale cyclonic modon as $p_{\text {bot }} \rightarrow 20$, particularly in the very deep fields ( $\mathrm{b}$ and d), compared with the flow in the shallow atmosphere simulations at this (and lower) horizontal resolution; but, the flow becomes much less zonal and is similar to that of the shallow atmosphere simulation at a higher horizontal resolution particularly in the region $p \lesssim 1$ (Skinner \& Cho 2021; Cho, Skinner \& Thrastarson 2021).

In the figure, modons are seen executing westward translations around the planet in the bulk, on a few to $\sim 20$ planet-day periods. But, there are pauses, reversals of direction and short period $(\lesssim$ day) oscillations superimposed on the bulk motion. This is due to the strong interaction with small-scale structures. Frequently, the modons break and reform at the end of their "lifecycles" (e.g. Fig. 8). However, while both types of modon feature quasi-periodic life-cycles, they generally display large differences in periodicity and phase between them - after the initial, formative period of $\sim 10$ planetary days.

In a typical life-cycle of the cyclonic modon, the modon first forms slightly westward of the sub-stellar point and initially translates eastward - consistent with its cyclonic character in the background of no, or very weak, motion. After reaching the sub-stellar point, the modon often "hangs" in this position for an extended pe- riod of time (up to $\sim 10$ planetary days). ${ }^{10}$ Near the sub-stellar point, the cyclonic modon generates and interacts with a very large number of small-scale storms of opposing signs to the cyclonic modon in the northern and southern hemispheres (Fig. 5b). This effectuates the meridional (as well as enhances the zonal) symmetry breaking, causing the modon to oscillate about the sub-stellar point by $\sim 10^{\circ}$ in longitude. This is concomitant with a decrease in the modon's size and strength as well as an increase in angular separation between the constituent cyclones, thus rendering the modon susceptible to further perturbations. This is a characteristic property of modons in the ageostrophic regime. After the extended pe-

10 In some cases, it can be as long as $\sim 100$ planetary days initially, if the zonal symmetry remains unbroken or only very mildly broken (e.g. when the forcing is more symmetric or dissipation steers the flow). 
(a)
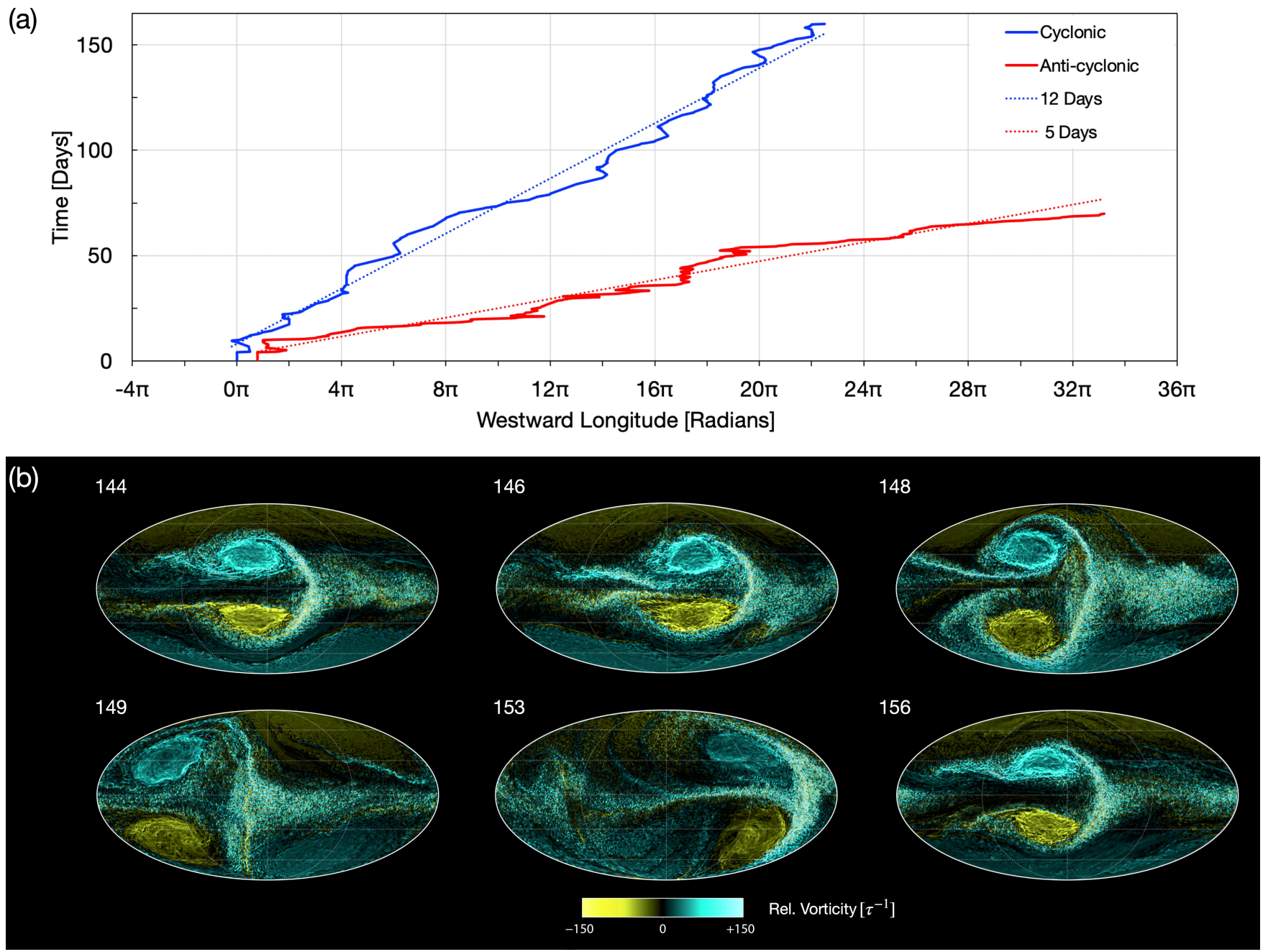

Figure 7. (a) Longitude position of the modon centroids (in radians) westward of the sub-stellar point, as a function of time $t=[0,125]$ and at $p=0.1$, from the simulation in Fig. 5. Blue and red lines correspond to the cyclonic and anticyclonic modon centroid trajectories, respectively. Dotted lines are constant westward migration rate fits with periods 12 and 5, as indicated in the legend. Both modons exhibit quasi-periodic life-cycles in which they translate chaotically westward around the planet in the bulk, undergoing multiple excursions about a smooth straight-line trajectory. On multiple occasions, modons pause at the day-side (night-side) and oscillate to either side of the sub-stellar (anti-stellar) point, before continuing on. Such behaviours are significant for observations. (b) The relative vorticity $(\zeta)$ field from the simulation shown in Fig. 5 at $p \sim 0.1$ and in Mollweide projection showing a single cycle of a modon's westward translation around the planet.

riod near the sub-stellar point, the modon moves westward, past the western terminator and quickly traverses the night-side to reach the eastern terminator in a nearly continuous motion. The traversal usually takes no longer than $\sim 5$ planetary days. Finally, at the eastern terminator, the modon's constituent cyclones uncouple and dissipate completely or move to high latitudes - whence the cycle ends. A new cyclonic modon subsequently forms on the day-side and the cycle begins again. In total, the cycle lasts for $\sim 15( \pm 4)$ planetary days, of which the modon generally occupies the day-side for $\sim 10$ planetary days.

While the anticyclonic modon's life-cycle is qualitatively similar to that the of the cyclonic modon, it is shifted in phase (longitudinal location) and has a considerably shorter migration period. The latter is because the anti-cyclonic modon is generally located nearer to the planet's poles and precesses only slightly off the polar axis at high-latitudes, rather than traversing the entire circumfer- ence of the planet near the equator. Ultimately, this is related to the asymmetry of the thermal forcing (recall that the night-side equilibrium temperature is uniform and lower than the day-side temperature). Consequently, the variability can be considerably different, depending on the latitude being viewed. This points to the importance of knowing the planet's obliquity (or inclination angle), when interpreting atmospheric variability. Further, note that the cyclonic and anti-cyclonic modon tracks are roughly anti-correlated in longitude in Fig. 7; for example, the anti-cyclonic modon forms on the night-side at the same time as the cyclonic modon, which forms on the day-side. Such coupled behaviour presents additional "targets" for current and future observations. 


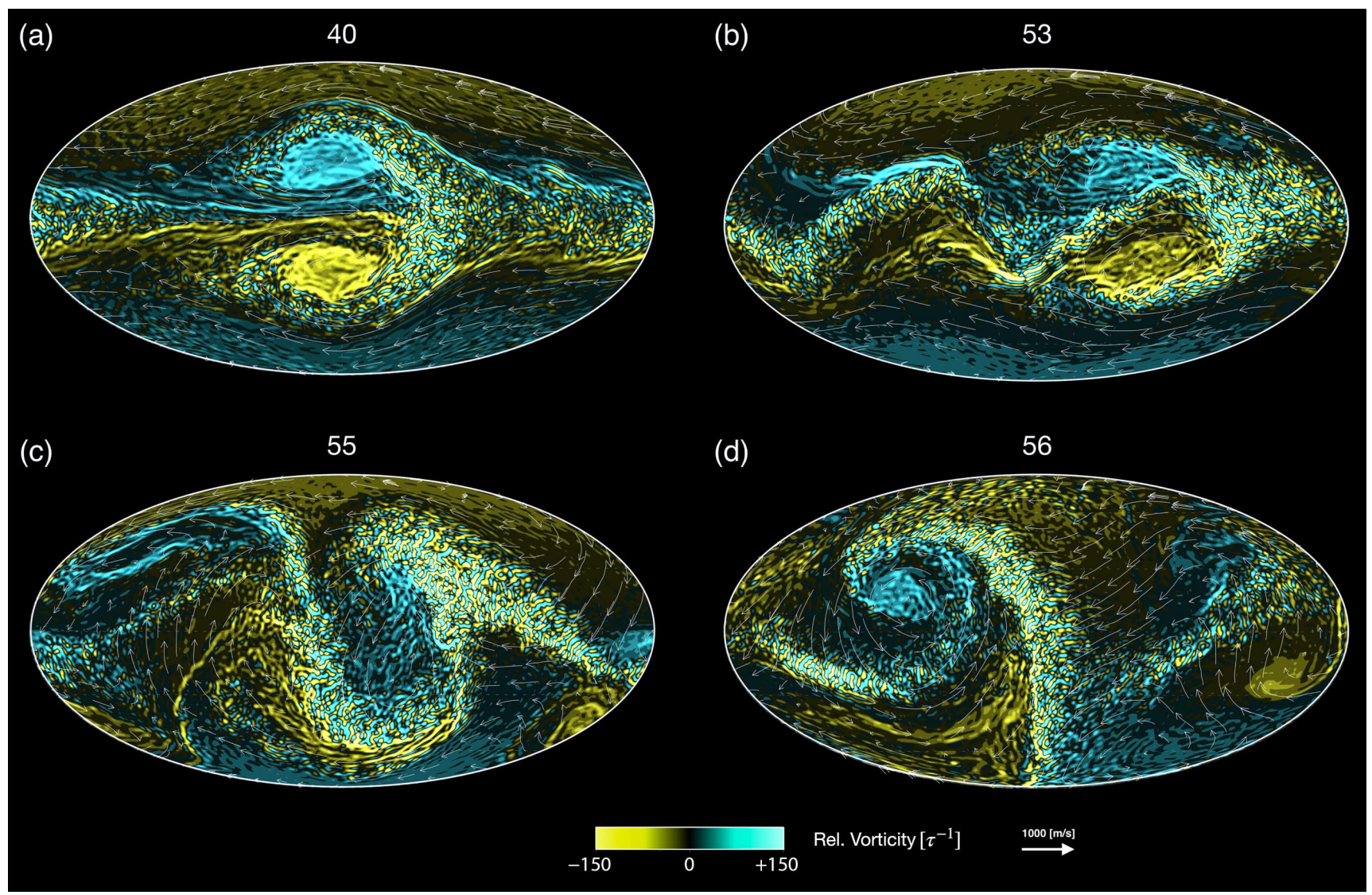

Figure 8. The $\zeta$ field, with overlaid winds (reference vector length is $1000 \mathrm{~m} \mathrm{~s}^{-1}$, equal to 3.025 in $R_{p} \tau^{-1}$ units) at $p=0.1$ from a T341L20 simulation, with $\mathfrak{p}=8, \nu_{16}=1.5 \times 10^{-43}, \Delta t=4 \times 10^{-5}$ and vertical range of $[0,0.1]$. The four frames illustrate the onset of a secondary equilibrium state that arises in the simulations. In this sequence, a modon breaks apart into fast moving, uncoupled cyclones; and, the atmosphere is rapidly mixed as a consequence. At $t=40$ a cyclonic modon, which has spent an extended period of time positioned at the sub-stellar point, migrates eastward towards the night-side rather than continuing on its usual westward direction (a). As the modon reaches the eastern terminator $(t=53)$, it encounters fast flow from the night-side and shears apart, inducing very high amplitude undulations of the equatorial jet (b). At $t=55$, both the equatorial jet and the cyclonic modon are converted into smallscale storms, which are transported westward around the planet by very high-amplitude Rossby waves (c). At $t=56$, the small-scale storms coalesce into large-scale cyclones and the polar vortices break up (d). The atmosphere undergoes a period of vigorous mixing before a new modon forms on the day-side. This sequence of patterns recurs quasi-periodically over the duration of the simulation.

The above discussion suggests a multitude of quasiequilibrium flow states ${ }^{11}$. These states are associated with distinct flow configurations and periodicities and lasts for extended periods of varying durations. Moreover, multiple transitions between the states within a single simulation occur as well. In general, the set of available states vary with $p$-levels; but, they are often correlated. The overall behaviour is generic across small variations in thermal forcing profile and initial flow condition. However, as expected, the behaviours is quantitatively different at lower resolution and even qualitatively different below T85 resolution. The latter is because simulations below the T85 resolution (particularly with $\mathfrak{p}<8$ ) produce modons (when they are able) that are not dynamic (Skinner \& Cho 2021).

Fig. 8 presents an example of a state transition that frequently occurs in the current setup. The four frames in the figure illustrate the key stages of a transition between two states known as "block-

11 We define "quasi-equilibrium" as a state that persists over a long duration - i.e. over a period much longer than $\max \left\{\tau_{\text {th }}, \tau_{\text {ad }}\right\}$, when $\tau_{\text {th }}<\infty$. ing patterns" - a "diffluent block" and an "omega block". ${ }^{12}$ In the figure, blocking actions rapidly mix and chaotically churn the atmosphere over a several-day time-scale. The transition is from the diffluent block to an omega block and eventually back to the diffluent block (often after a complete dispersal of the original modon, as shown). We note that the modon in this example had previously completed many traversals of the planet prior to this breakup. Instead of continuing its westward translation, this modon translates eastward across the sub-stellar point close to the eastern terminator (Fig. 8a), where it starts to tilt in the counter-clockwise direction (Fig. 8b). In the latter, the shearing modon disrupts the equatorial jet, generating many hundreds of small-scale storms in the process. After one to two planetary days, the modon is nearly sideways, fully into the omega state (Fig. 8c). At this point, the small-scale storms generated are carried westward from the night-side to the

12 In meteorology, blocks are large-scale weather patterns that are nearly stationary and effectively block and redirect flows (Rex 1950; Woolings et al. 2018). 
day-side by (very) high-amplitude Rossby waves (e.g. undulation of the equatorial jet). Fig. 8d shows the resulting flow pattern after the small scale-storms have coalesced into a large, energetic cyclone that veers off northwest, into the night-side. Unlike the modons, these uncoupled cyclones rapidly traverse the planet, inducing further vigorous mixing. The whole process causes the atmosphere to be much more homogenised. Note that studies have demonstrated that higher resolution improves the representation of the blocking in climate models (of the Earth), when compared with observations (e.g. Anstey et al. 2013). Thus, we reiterate here one of our major points in this paper: higher resolution, than employed in the past, is necessary to capture the atmospheric states of hot extrasolar planets accurately.

\subsection{Dynamic Temperature Redistribution}

In this subsection, we discuss more directly the effects of modons on the temperature distribution of the atmosphere - and, more broadly, observable signatures produced by them. Our discussion here centres mainly on the shallow atmosphere simulations; however, we also present and discuss deep atmosphere simulations. One reason for the shallow atmosphere focus is that there is little qualitative difference between simulations with $p_{\text {bot }} \in\{0.1,1.0\}$ (recall that the applied thermal forcing does not extend to $p \geqslant 1$ region). Another important reason is that adequately resolving - and choosing - the vertical range of the atmosphere (including choosing the location of $p_{\text {bot }}$ ) is still uncertain and computationally challenging in $p_{\text {bot }} \gg 1$ cases (Skinner \& Cho 2021). Qualitatively, the general flow pattern changes monotonically as $p_{\text {bot }}$ increases from 0.1 to 1.0 ; and, the modons become weaker and wider with the equatorial jet becoming more zonal, as $p_{\text {bot }} \rightarrow 1$. We have verified that this general picture does not change qualitatively as $p_{\text {bot }} \rightarrow 20$, with up to T341L200 resolution. Deep atmosphere simulations are discussed below. Note that the qualitatively robust general behaviour of the modons is due to the strongly barotropic quality of the flow. This is intimately related to the setup employed.

Fig. 9 shows the instantaneous temperature anomaly field, $T_{a}(\lambda, \phi)=T-T_{\mathrm{eq}}$, at $p=5 \times 10^{-3}$. The $T_{a}$-fields are chosen from the T341L20 simulation shown in Fig. 8, when the modons are not in one of the "breakup" cycles: the fields in a "quiescent" cycle are presented. The fields at $p=0.1$, corresponding to the bottom of the shallow atmosphere simulation, match very closely with the presented fields because of the strong barotropic character of the flow. The $T_{a}$-field field reveals the regions of heating $\left(T_{a}<0\right)$ and cooling $\left(T_{a}>0\right)$, or absorbing and emitting, respectively. For greater lucidity, the fields are shown in orthographic projection, centred on the anti-stellar and sub-stellar points, illustrating the night-side (NS) and day-side (DS) discs separately. Note that, in the absence of dynamics, $T_{a}=0$ globally. A primary aim of this work is to demonstrate clearly that the flow induces a significant deviation from this - even when $\tau_{\text {th }} \ll \tau_{s}$ (Fig. 1). This is in contrast to what is reported in many previous studies (e.g. Cooper \& Showman 2005; Showman et al. 2008; Liu \& Showman 2013). Another major aim is to clearly demonstrate that heat is transported mainly by eddies (storms) rather than the equatorial jet, as is often asserted.

As can be seen in Fig. 9, the local emission and absorption are strongly dependent on the location, size and strength of both modons. For example, at $t=91$ on the day-side, temperature is localised to two "hot spots" of $T \approx T_{\text {eq }}$, where each of the "spot" is really one of the modon's cyclones sequestering hot air in its (extended) core (Fig. 9a). Importantly, the sequestered regions are nei- (a)

91
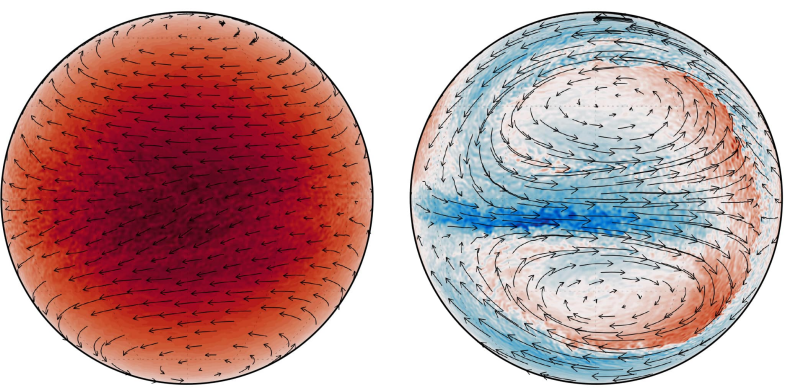

(b)

93
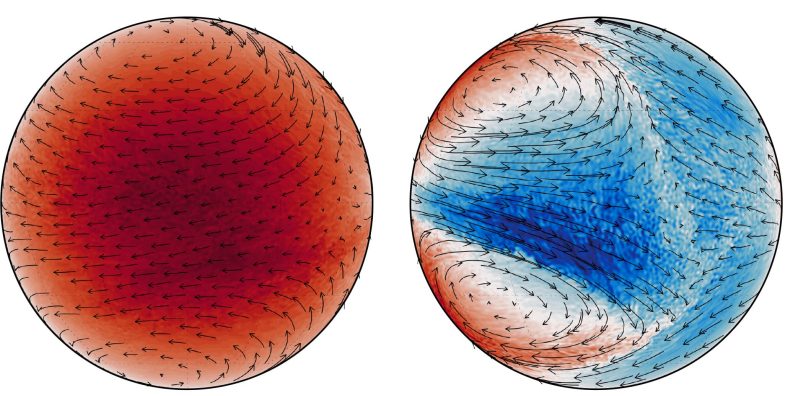

(c)

95
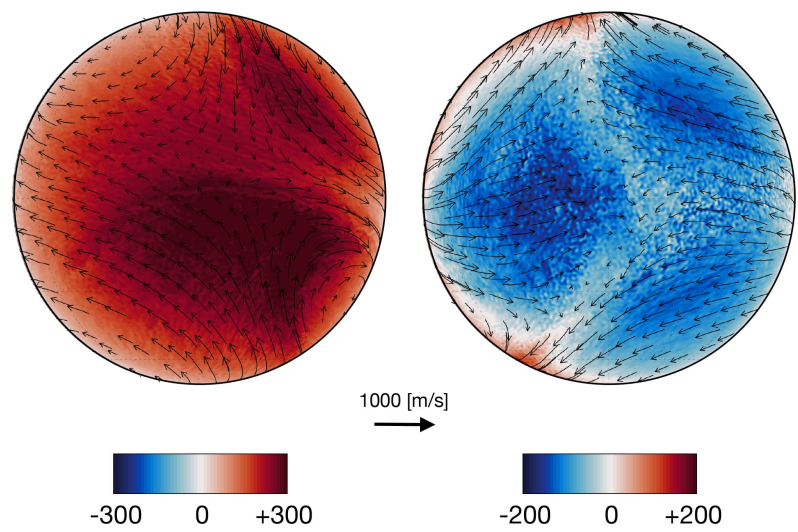

$\stackrel{1000[\mathrm{~m} / \mathrm{s}]}{\longrightarrow}$

Temp. Anomaly $[\mathrm{K}]$

Figure 9. Temperature anomaly field, $T_{a}(\lambda, \phi, p)=T-T_{\mathrm{eq}}$, with the winds overlaid: fields from the simulation in Fig. 8 are shown at $p=$ $5 \times 10^{-3}$ at the indicated times (however, the modons are in their "quiescent" cycle, in contrast that in Fig. 8). Left and right columns show the night-side (NS) and day-side (DS) discs, respectively; and, the projection is orthographic. Red and blue colours indicate regions that are cooling ( $T>T_{\text {eq }}$ ) and heating $\left(T<T_{\text {eq }}\right)$, respectively: hence, they indicate regions which are absorbing and emitting, respectively. (a) Day-side emission is localised in a narrow arc at the cyclonic modon's eastern periphery; the modon's two cyclones sequester hot air in their cores, but they are not emitting $\left(T_{\mathrm{eq}} \approx 0\right)$, due to the short relaxation time. The day-side equatorial jet is strongly absorbing at this time and does not "transport heat", but rather cold temperature (see also $t=93$, DS). The anti-stellar point is generally emitting because the equatorial jet and polar modon directs hot flow from the day-side. (b) When the cyclonic modon migrates to the western terminator, hot air from the day-side is transported to the night-side, around the periphery of the cyclonic modon via the poles. (c) A local patch of strong emission on the night-side $\left(T_{a} \approx 100 \mathrm{~K}\right)$ is generated. Since the modons migrate periodically around the planet, the simulations exhibit periodic peaks in emission on the day and night sides. 
ther emitting nor absorbing thermal radiation since $T_{a} \approx 0$; hence, the dynamics is also thermally unforced in these large regions. Both the limb and equatorial jet of the day-side disc are strongly absorbing, where $T_{a} \approx-125 \mathrm{~K}$. In contrast, the night-side is emitting in a wide area near the anti-stellar point with $T_{a} \approx+40 \mathrm{~K}$. At $t=93$, as the modon migrates from the sub-stellar point to the western terminator, hot air is transported to the night-side towards the higher latitudes (Fig. 9b). Consequently, the day-side and nightside are now in absorption and emission, respectively. At $t=95$, as the cyclonic modon emerges on the night-side, the westward jet between the anti-cyclonic modon begins to intensify at the equator and the meridional flow on the western flanks of the cyclones converges to the anti-stellar point, transporting hot air over the high latitudes from the day-side to the night side (Fig. 9c). This results in a more continuously heated (i.e. emitting) night-side, with periodic bursts of strong emission. The period of oscillations (of both emission and absorption) is about 10 to 15 planetary days, as described above (Fig. 7).

Fig. 10 shows the actual redistribution of temperature $T$ by the cyclonic modon over the full traversal around the planet. Fig. 10a shows the instantaneous temperature fields with overlaid wind vectors in Mollweide projection at $p=0.1$. Six frames in the interval, $t=[93,105]$, illustrate various stages in this particular type of thermal cycle. We reiterate that, in the absence of dynamics, the $T$ field shown would resemble a simple circular patch of "hot spot" centered at the sub-stellar point, instead of the two disjointed patches associated with the modon's cyclones (as was seen in Fig. 9). In Fig. 10b, the disc-integrated flux time-series (also at $p=0.1$ ), encompassing the frames in Fig. 10a, are presented for four of the planet's key locations: sub-stellar point (SS), anti-stellar point (AS), eastern terminator (ET) and western terminator (WT). At each of these locations, the local "radiative equilibrium flux" (i.e. $\sigma T^{4}$ ) from Fig. 10a is averaged over the disc, weighted by a cosine factor to account for the spherical geometry. ${ }^{13}$ The flux is normalised by the the initial flux based on $T_{\text {ref }}(p)$. In the absence of additional physical parameterisations (e.g. radiative transfer, cloud, and species distributions) which can be added for increased physical realism, $\sigma T^{4}$ is an adequate measure of the equilibrium flux. A more realistic flux using a radiative transfer model will be reported elsewhere.

The sequence of frames shown in Fig. 10a follows the modon from its formation near the sub-stellar point $(t=93)$, to its cooling $(t=\{98,100\})$, to breakup and diffusion $(t=102)$ and the subsequent reformation and re-heating $(t=105)$. Overall, the modon's periodic motion in this example redistributes planetary-scale regions of hot and cold air zonally (both westward) with $\sim 12$-day period. The cyclonic modon initially sequesters heat (hot temperature) at the day-side in each of its cyclones and form two distinct hot patches straddling the equator (separated by a narrow, meandering, cold jet between them). As already discussed, meridional redistribution occurs as well $(t=\{100,102\})$. Note that, the cyclonic modon transports cold air from the night-side $(t=102)$; at the same time, the anti-cyclonic modon can also be seen transporting hot air. Shortly after, the once cold cyclonic modon has warmed up upon reaching the sub-stellar point $(t=105)$.

The modon's rearrangement of temperature leads to discernible signatures in the time-series of the disc-integrated flux at

13 From hereon, we shall simply refer to this quantity as "temperature flux", or often just "flux", as in Skinner \& Cho (2021) and Cho, Skinner \& Thrastarson (2021). each of the four chosen locations, as shown in Fig. 10b. In general, the SS flux (at the peak of the secondary eclipse) is the largest and the AS flux (at the peak of the primary eclipse) is the smallest of the four fluxes. But, the variations in each flux is such that SS flux can be lower than the ET flux and the ET flux can be lower than the WT flux, at different times and with different periods. Fig. 10a and Fig. 10b both show that the hottest (coldest) region can be located over a broad range of longitude, and on either side of the sub-stellar (anti-stellar) point.

Fig. 11 shows the flux time-series at $p=0.1$ over a long duration from a simulation at T341L20 resolution with $\mathfrak{p}=8$, $\nu_{16}=1.5 \times 10^{-43}$ and $\Delta t=4 \times 10^{-5}$; this is the simulation presented in Fig. 8. Top and bottom frames in Fig. 11 show the fluxes at the sub-stellar and anti-stellar points (top) and the fluxes at the two terminators (bottom), respectively. The generic flux variability seen in Fig. 10b, associated with the modon's generally cyclic behaviour, persists for 1500 planetary days; hence, the simulations and behaviours presented in this paper are assumed to be in quasi-equilibration. We note that this simulation is also numerically converged (see Skinner \& Cho 2021). Although not quantitatively comparable, transitions to several different quasi-equilibrium states are expected, based on extremely long-duration (over 80,000 planetary days) simulations at much lower resolution (Thrastarson 2011) - hence, the "quasi" qualification (because of the transitions). Roughly, two states are readily apparent in the figure: "active" state and "quiet" state, based on period and/or amplitude. Note that these temperature time-series track the flow's global eddy kinetic energy (cf. Fig. A1 in the Appendix). The quiet regions in the fluxes correspond to the "omega blocking" quadrupolar vortex configuration which rapidly mixes the hot and cold regions more evenly though out the flow. The bursts in the flux are caused by the slower moving modons, which do not mix the atmosphere as quickly and carry the heat (hot temperature) as they migrate. The slower moving modon traps air on the day-side for longer period, causing the flux amplitude to be higher on the day side. This is also why the SS and ET flux variances are high, compared to the other fluxes, during the modon formation phase (see also Fig. 10b).

Fig. 12 shows the power spectra of the disc-averaged flux time-series in Fig. 11. Information such as this (from converged simulations) can aid in planning or scheduling observations (Cho, Skinner \& Thrastarson 2021; Skinner \& Cho 2021). The spectra are computed from the time-series data between days 25 and 1500 to neglect variability associated with the simulation's initial rampup phase. The power spectra are normalised such that the variance of the de-trended series is equal to $\sum_{j} S(j) \mathrm{d} f$, where $S(j)$ is the time-series data with $j$ samples and $\mathrm{d} f$ is the frequency spacing (4 per day in this case).

All of the power spectra in Fig. 12 are broad and "rich", with many peaks over a wide range of periods. In general, all the spectra contain peaks at $\sim 8$-planetary days and $\sim 15$-planetary days. The spectra also contain peaks of shorter period ( $\sim 4$-planetary days) and longer period ( $\sim 25$-planetary days), including those of very long periods (i.e. $\gtrsim 100$-planetary days). All of these periods can be directly attributed to flow induced temperature field evolutions discussed in this work. Further, as can be surmised from data shown below, the corresponding four spectra at high $p$-levels in the deep atmosphere simulations are markedly different than those in Fig. 12: the spectra from the high $p$-levels are not broad and contain only few peaks (see Cho, Skinner \& Thrastarson 2021). These levels, of course, do not exist in shallow atmosphere simulations. The corresponding four spectra at high $p$-levels are also essentially identical to each other, showing that the life-cycles in the deep re- 
(a)
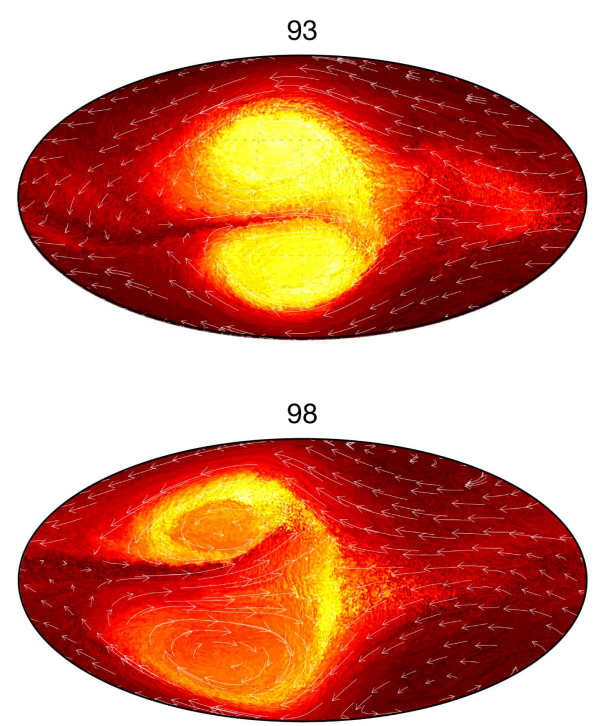

102

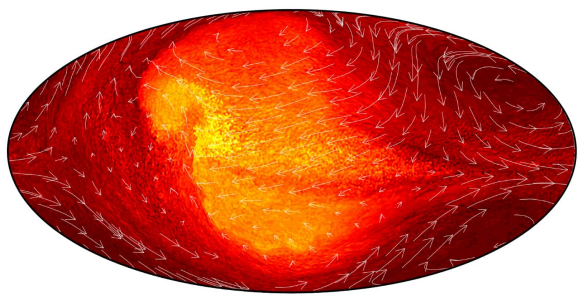

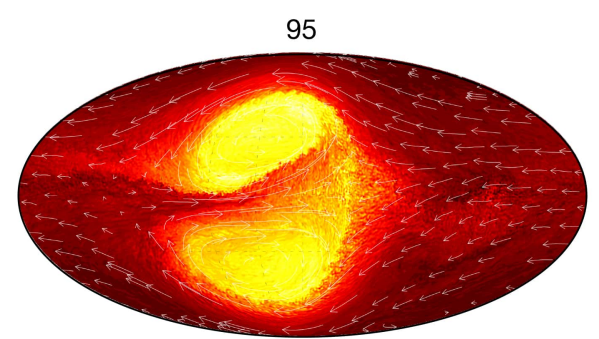

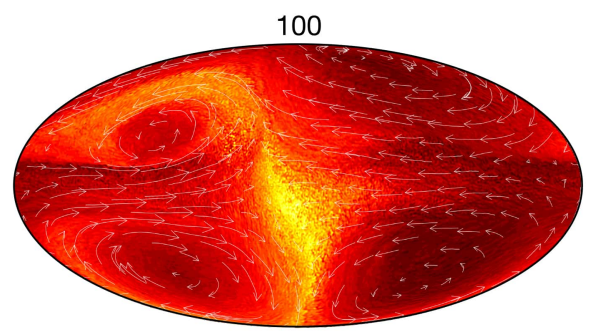

105

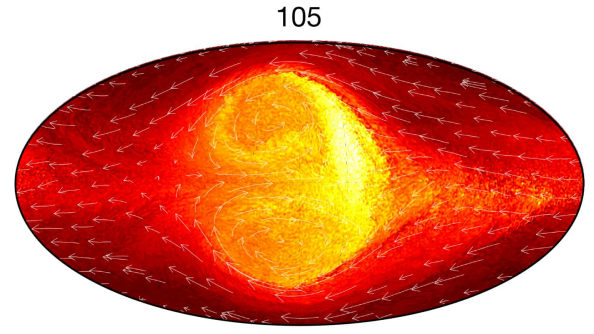

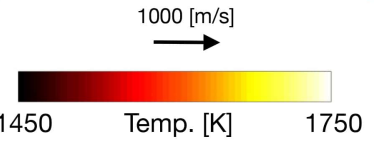

(b)

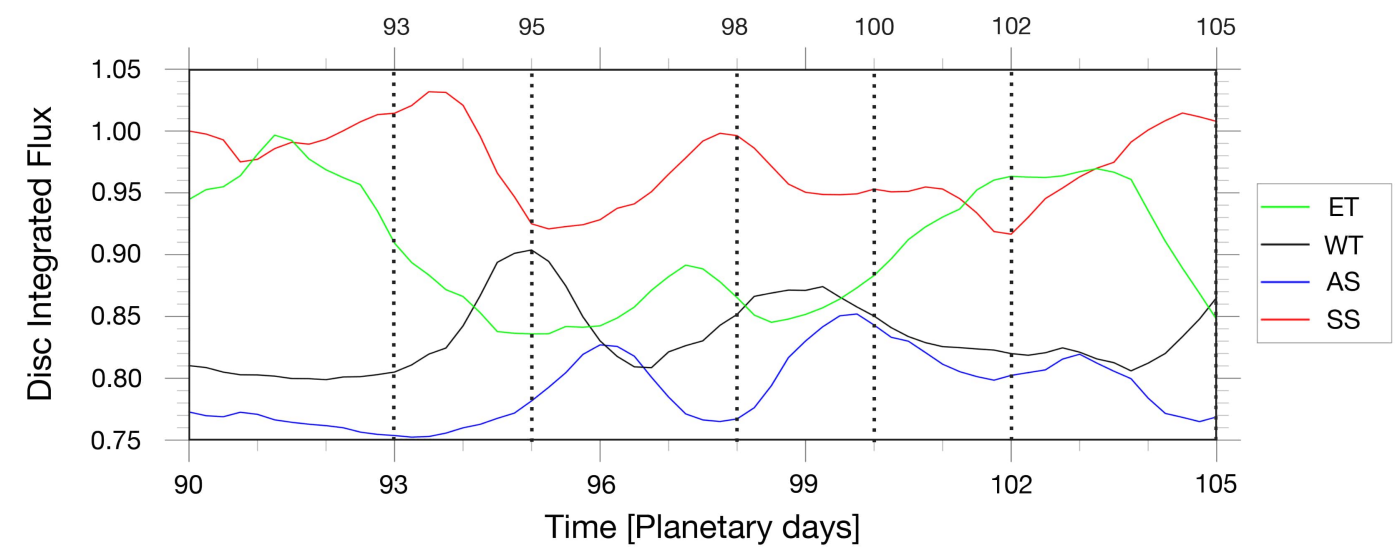

Figure 10. The cyclonic modon's periodic migration zonally redistributes planetary-scale regions of hot (and cold) flow with $\sim 12$ planetary day periodicity, and latitudinally mixes the temperature distribution of the atmosphere. (a) Instantaneous temperature fields (in units of $K$ ) with overlaid winds at the $p=0.1$ level. Time frames $t=[93,105]$ illustrate key stages in the life-cycle of a cyclonic modon and the corresponding global temperature distribution. At $t=93$, a modon forms near the sub-stellar point and migrates westward around the planet over a 12-day period, in which it mixes and redistributes hot (cold) regions of the flow. At $t=102$, the cyclonic modon dissipates at the end of its life-cycle and subsequently reforms at $t=103$. The modon's effect on the global temperature distribution produces discernible signatures in the time-series of normalised disc-integrated flux, $T / T_{\text {ref }}$ (b) at each of the planet's key locations: eastern terminator (ET), western terminator (WT), anti-stellar point (AS), and sub-stellar point (SS). In general, the SS flux is the largest and the AS flux is the smallest of the four fluxes - as expected, given the short $\tau_{\mathrm{th}}$. However, the variations in each flux is such that SS flux can be lower than the ET flux and the ET flux can be lower than the WT flux, at different times and with different periods. Both (a) and (b) show that the hottest (coldest) region can be located over a broad range of longitude and on either side of the sub-stellar (anti-stellar) point. 
T341
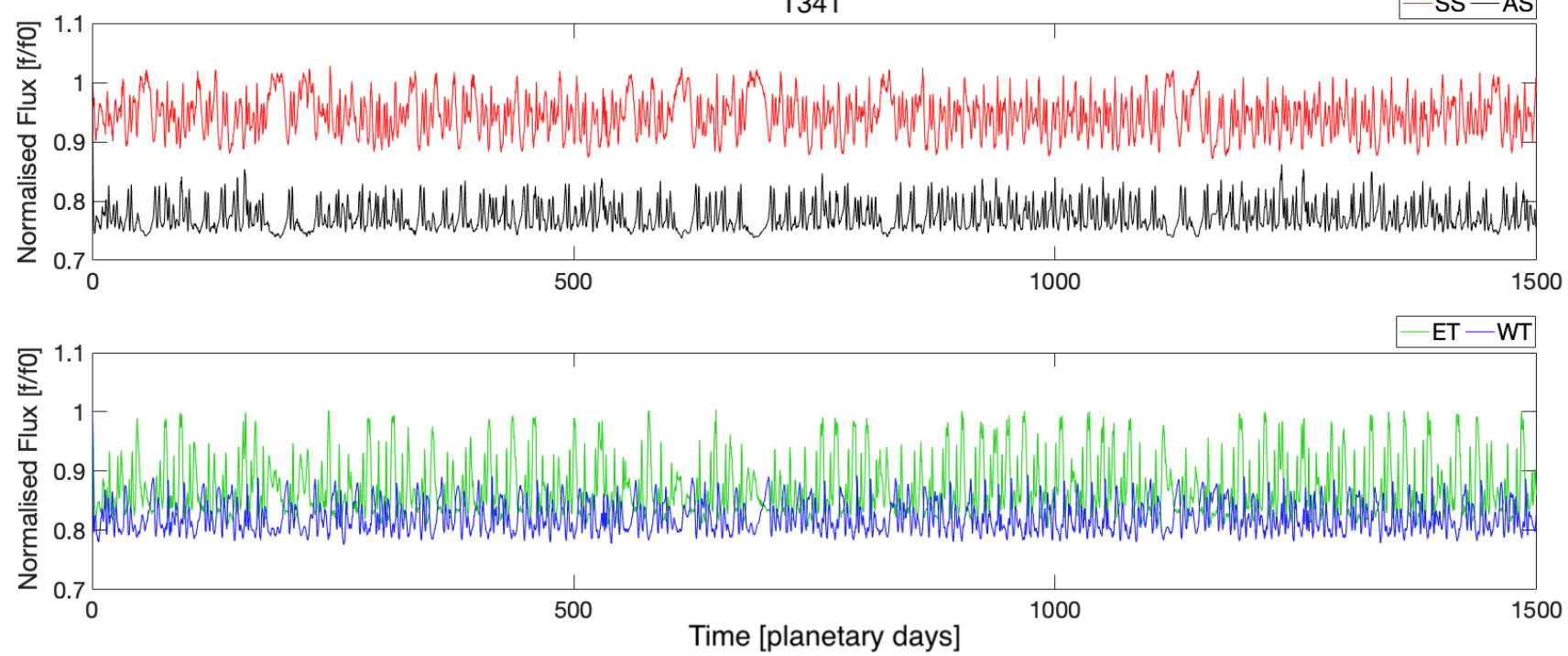

Figure 11. Long duration time-series of normalised disc-integrated flux at $p=0.1$ for a simulation at T341L20 resolution with $\mathfrak{p}=8, \nu_{16}=1.5 \times 10^{-43}$ and $\Delta t=4 \times 10^{-5}$. Top and bottom panels show normalised disc-integrated flux at the sub-stellar (SS) and anti-stellar (AS) points and the eastern terminator (ET) and western terminator (WT), respectively. Quasi-periodic variability associated with the modon's behaviour (e.g. Fig. 10) persists for 1500 planetary days; hence, the simulations and behaviours presented above are assumed to be equilibrated over the duration shown. The time-series behaviours can be directly related to temperature redistribution by the modons.
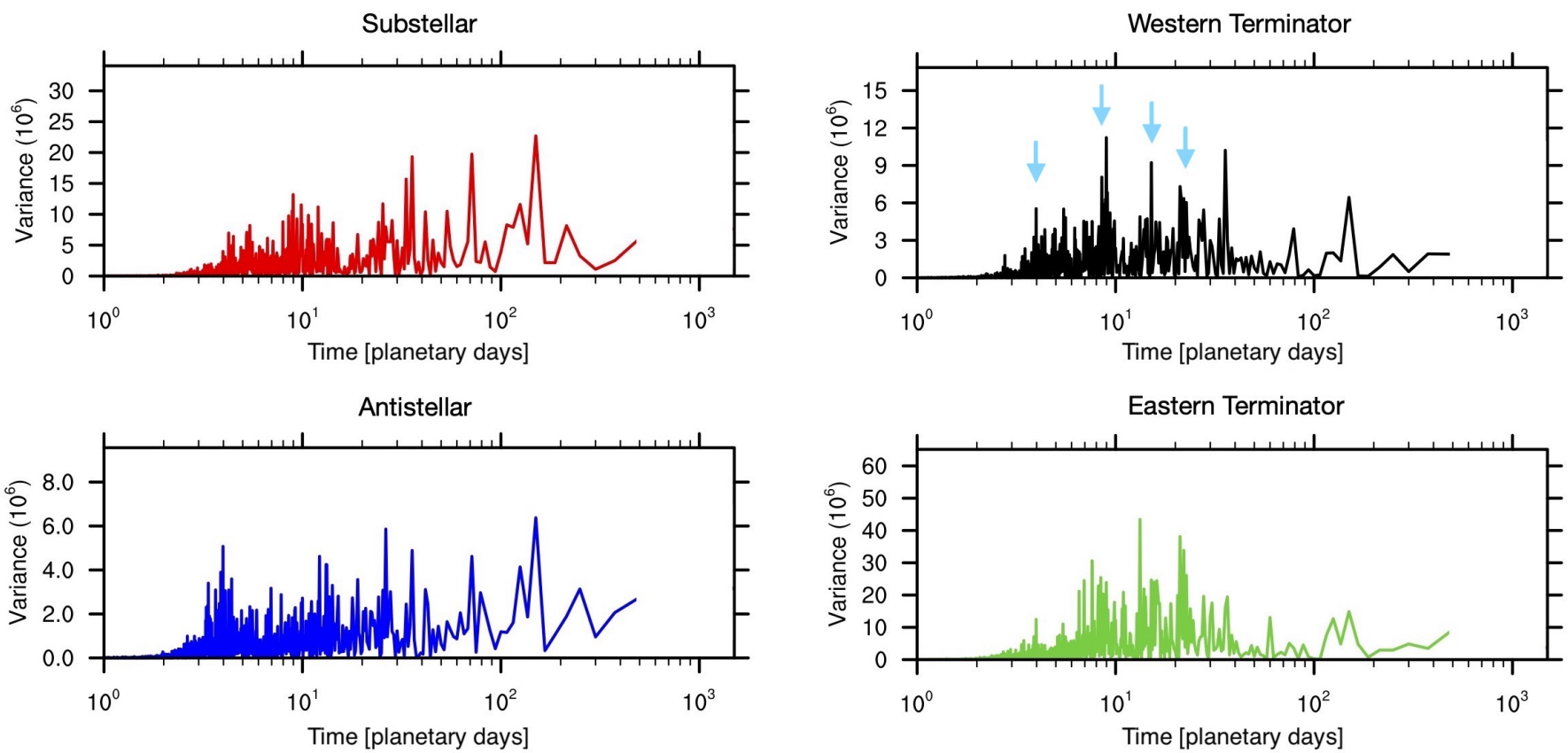

Figure 12. Power spectrum normalised by the variance of the disc-averaged temperature flux time-series in Fig. 11. All four spectra are broad and "rich" (i.e. many peaks over a wide range of periods). All of the spectra generally contain peaks at $\sim 8$-planetary days and $\sim 15$-planetary days, with peaks at shorter period ( $\sim 4$-planetary days) and longer period $(\sim 25$-planetary days): these are labelled in the upper right frame. The corresponding four spectra at high $p$-levels in the deep atmosphere simulations (not shown) are markedly different - as might be expected from Fig. 14; see also Cho, Skinner \& Thrastarson (2021), Fig. 4. Spectra in the deep region are not broad and contain only few peaks. They are also essentially identical to each other, showing that the life-cycles in the deep region are much more regular and differ only in phase. Many peaks can be directly related to the temperature redistribution by the modons, as in Fig. 11 . 
(a)

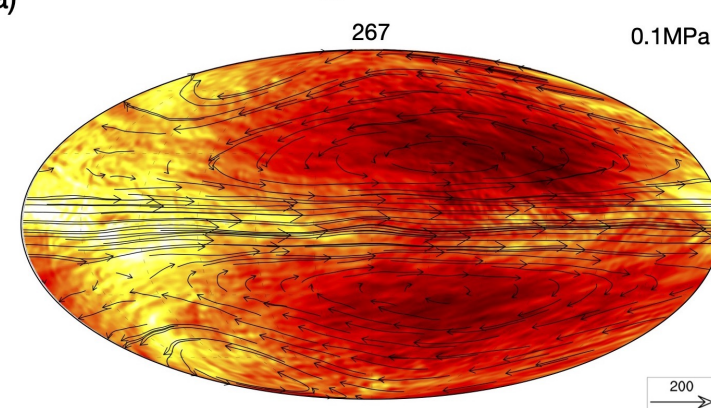

(b)

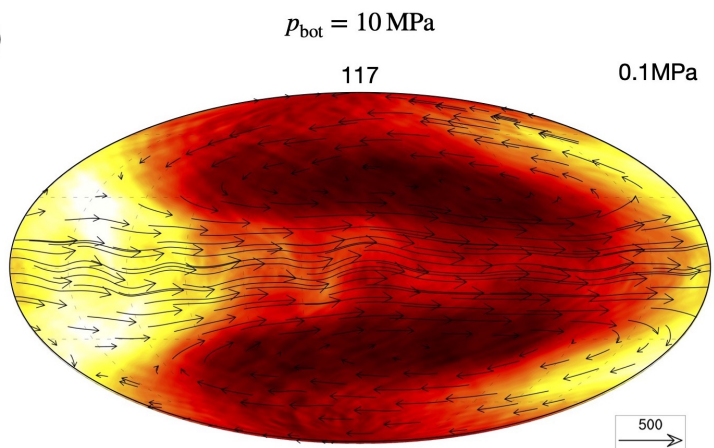

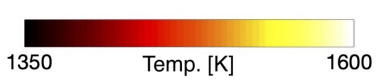

(c)

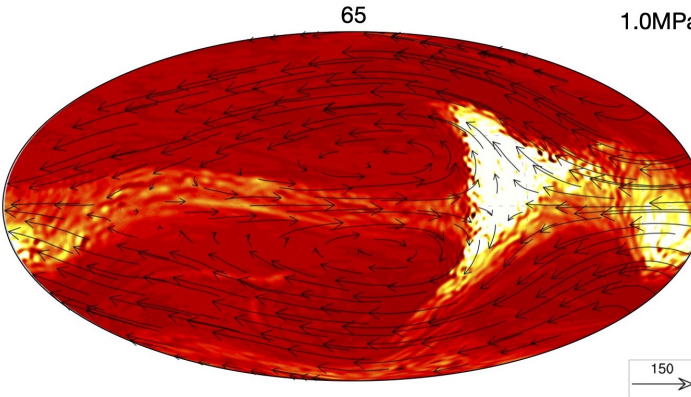

(d)

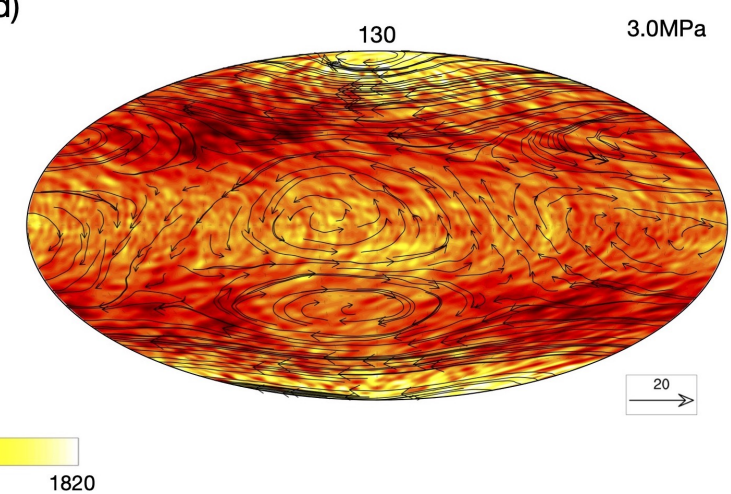

Figure 13. Temperature and wind (black arrows) fields centred on the night-side, from two T170L200 deep atmosphere simulations with $p_{\text {bot }} \in\{1,10\}$; $\mathfrak{p}=8, \Delta t=8.0 \times 10^{-5}$ and $\nu_{16}=10^{-38}$. Time is indicated above each frame. The reference vector length and temperature range are adjusted to accommodate the different background (flow and temperature) conditions. The four frames illustrate modons occurring at different times and $p$-levels (as labelled) throughout both simulations. As in the shallow atmosphere simulations (cf. Fig. 10), modons mix and redistribute hot (and cold) regions over the planet - including in region that is not subject to direct thermal forcing. All of the modons at the centre of each frame are cyclonic modons.

gions are much more regular and differ only in phase, since phase differences are not detected by a power spectrum (Cho, Skinner \& Thrastarson 2021).

Fig. 13 presents the temperature $T$ and wind fields of two deep atmosphere simulations which include $p$-levels that are not thermally forced (i.e. $p \geqslant 1$ ). These simulations are identical in setup, with T170L200 resolution, $\mathfrak{p}=8, \nu_{16}=10^{-38}$ and $\Delta t=8 \times 10^{-5}$ - except for the different values of $p_{\text {bot }}=\{1,10\}$. Note that the two simulations presented are not numerically converged (see Skinner \& Cho 2021); however, they are shown here are for illustrative purposes - in order to demonstrate that modons form in simulations with different $p_{\text {bot }}$ values, at a variety of $p$-levels and simulation times. These modons are still expected to form in converged (i.e. $\gtrsim$ T341L4000) deep atmosphere simulations. Moreover, the simulations presented are still greater in resolution than nearly all past extrasolar planet studies to date. As in Fig. 10a, the $T$ field is displayed in the Mollweide projection, but it is now centred on the anti-stellar point to show modons located on the planet's nightside. Note also, the reference vector lengths in the different frames are adjusted to accommodate the different background (flow and temperature) conditions at the $p$ levels presented.

As can be seen in Fig. 13, modons occur throughout the modelled atmospheres - independent of the location of the domain bottom $p_{\text {bot. }}$. As just alluded to, for a quantitatively accurate assess- ment (e.g. detailed comparison with converged deep simulations), a sufficient number of vertical levels spanning the domain range is required. However, the modons in Fig. 13 also redistribute hot and cold patches of air as they translate around the planet, periodically breaking and reforming into several different configurations of vortices - as in the shallow atmosphere simulations (cf. Fig 10). Frames (a) and (b) shows explicitly that cyclonic modons also sequester and transport cold air. Note that the equatorial jet, which separate the cyclones of the modon, is warmer than the imposed $T_{\text {eq }}$ in both frames. Frames (c) and (d) show that modons, which form in $p$-regions not directly forced, transport and redistribute temperature. Not surprisingly, in these frames modons induce hottest patches of air to be at the night-side - and even at the poles (Fig. 13d). Such features are significant because, although much lower in amplitude than in the lower $p$-levels, fluxes can still come from the higher $p$-levels shown.

Fig. 14 shows the corresponding flux time-series at several key locations on the planet for the simulations presented in Fig. 13 in the time interval $t=[0,300]$. As in Fig. 13, left and right columns correspond to simulations where the bottom of the domain $p_{\text {bot }}$ is placed at 1 and 10, respectively. Top and bottom rows show the fluxes in the radiatively forced region (Fig. 14a and Fig. 14b) and below it (Fig. 14c and Fig. 14d), respectively. The figure shows that the generic behaviour seen in the shallow atmosphere simu- 
(a)

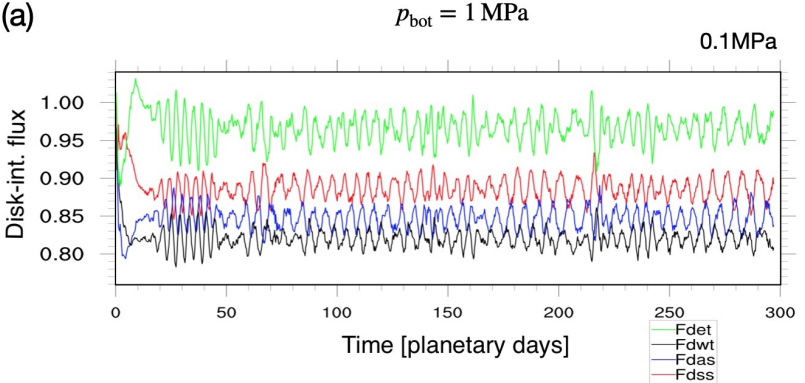

(c)

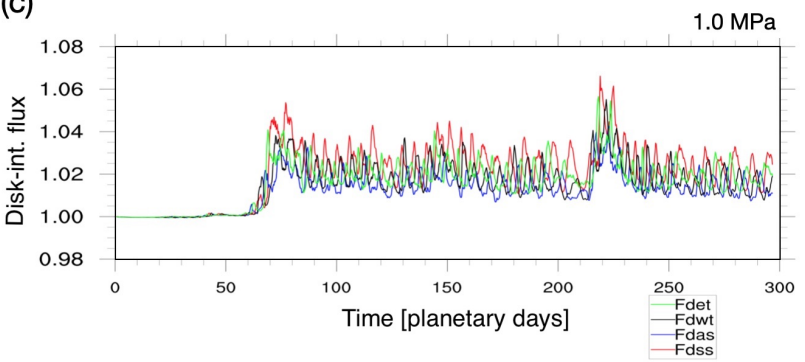

(b)

$p_{\text {bot }}=10 \mathrm{MPa}$

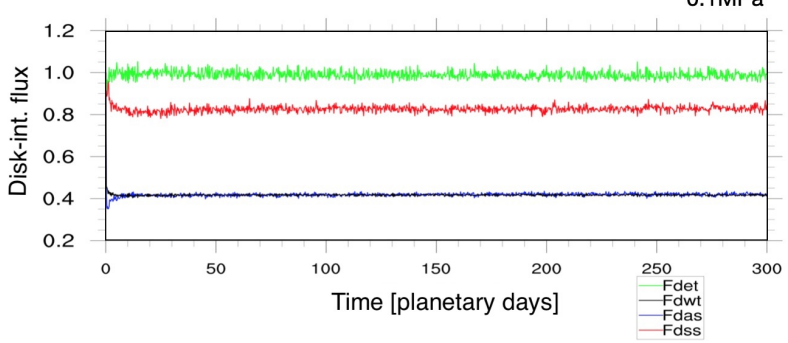

(d)

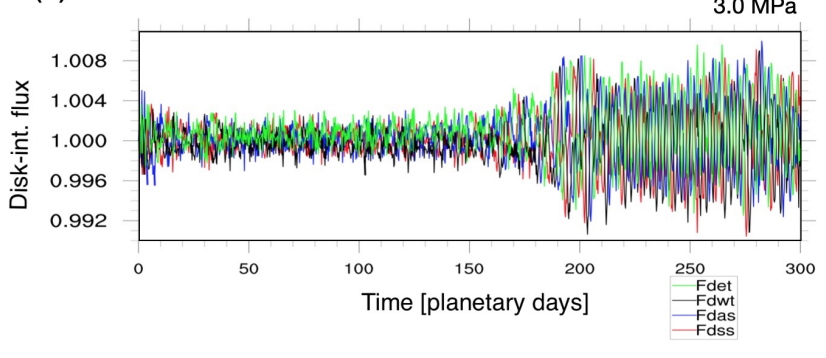

Figure 14. Disc-integrated temperature flux for the simulations presented in Fig. 6. Similarly to the shallow atmosphere simulations, the modon's effect on the global temperature distribution produces periodic signatures in the time-series of the disc-integrated fluxes at each of the planet's key locations and for different values of $p_{\text {bot }}$ (here $\{1,10\}$ ). In general, the flux variations are largest near the top of the atmosphere however periodic signals associated with the modons movements also occur at deeper levels. Quantitatively, the time-series behaviours are different compared to that in the shallow atmosphere (Fig. 11), consistent with the difference in the flows (cf. Figs. 5 and 13); however, features such as variability, quasi-equilibration and multiple states within and across $p$-levels are characteristic of both shallow and deep atmospheres. Strong vertical coupling is also evident in (a) and (c) - e.g. at $t \approx 60,165$ and 215 (see text).

lations (e.g. variabilities at multiple time-scales, state transitions and reduction in flux amplitude with $p$ ), also occurs in the deep atmospheres. Again, these behaviours are directly attributable to the behaviour of modons. The dynamism, however, requires that both the vertical and horizontal resolutions are sufficient to capture the modons with reasonable accuracy.

More quantitatively, the signals are the strongest at the eastern terminator location at $p=0.1$. This is in contrast with the fluxes from the shallow atmosphere simulation (cf. Fig. 11), which generally exhibits the highest amplitude signals at the sub-stellar location. Hence the signal is eastward shifted in the bulk (Fig. 14a); and, the shift is more pronounced with higher $p_{\text {bot }}$ value (Fig. 14b). This is due to the lower horizontal resolution, as well as inadequate vertical resolution for the $p_{\text {bot }}$ values (Skinner and Cho, in prep.). As discussed above, a robust $p_{\text {bot }}=10$ simulation requires $\sim 4000 p$-levels at T341 resolution. Qualitatively, a common feature in the deep atmosphere fluxes is a sudden jump in amplitude (e.g. at $t \sim 70$ in Fig. $14 \mathrm{c}$ and $t \sim 190$ in Fig. 14d). And, the jumps are generally correlated with sudden changes at $p=0.1$ (Cho, Skinner \& Thrastarson 2021). Such behaviour suggests the importance of properly resolving the directly forced $p$-region in deep atmosphere simulations and the utility of accurately modelling shallow atmospheres as well, since high horizontal resolution is still necessary even if high vertical resolution is achieved.

\section{CONCLUSIONS}

In this paper, we have summarised the results from a large set of high-resolution (up to T682L20 and T341L200), focusing on numerically converged simulations aimed at carefully studying the dynamics in a tidally synchronised extrasolar planet atmosphere.
We have found that a generic solution in the implemented setup is a pair of "oppositely-signed" (cyclonic and anti-cyclonic) planetaryscale modons. Note that, in general, the cyclonic modon is stronger than the anti-cyclonic modon; hence, the former tends to exert a stronger influence on the overall flow (and temperature redistribution) - although both modons are important. Both modons are also strongly barotropic and form at a variety of depths down to the $p=10 \mathrm{MPa}$ level with different vertical extents, depending on the location of the domain bottom level $p_{\text {bot }}$ (Skinner \& Cho 2021).

The modons in synchronised atmospheres are highly dynamic and feature a complex set of non-linear motions. Such behaviours have not been previously reported. Crucially, the complex motions are a result of non-linear interactions with energetic small-scale eddies and waves, which are not accurately captured in low-resolution and/or low-order dissipation simulations. For example, in our highresolution simulations with high-order dissipation, we have found that the modons are surrounded by a very large number of smallscale vortices and waves that develop in response to the modons' attempt to adjust in the ageostrophic flow environment. Ageostrophy is a generic characteristic of hot synchronised planet atmospheres. Thus, these atmospheres are highly turbulent, which is laterally extremely anisotropic (i.e. very "patchy" level-wise). The quasiperiodic nature of the flows also means the turbulence is robustly maintained overall, as the atmosphere is repeatedly stirred.

Broadly, our results present several significant implications for current and future observations of extrasolar planet atmospheres. In particular, we find that the atmospheres exhibit multiple equilibrium states, associated with the different cyclic behaviour of modons. The states produce temperature flux signatures at different locations over the planet. In different life-cycles, the modons undergo changes in direction, size and strength. The cyclonic and 
anticyclonic modons generally behave differently. In particular, the cyclonic modon typically dissipates completely near the eastern terminator before a new modon forms and the cycle repeats, whereas the anti-cyclonic modon often separates and the constituent anticyclones move towards their respective poles. Such motions result in vigorously mixing of temperature (and active species) in the atmosphere on the planetary-scale - by storms, rather than by a zonal equatorial jet.

\section{ACKNOWLEDGEMENTS}

The authors thank Michael E. McIntyre, Heidar Th. Thrastarson and Inna Polichtchouk for helpful discussions. We are grateful for the hospitality of James Stone and the Department of Astrophysical Sciences, Princeton University, where some of this work was completed. J.W.S. is supported by UK's Science and Technology Facilities Council research studentship. We thank the referee for the comments.

\section{DATA AVAILABILITY}

The data underlying this article will be shared on reasonable request to the corresponding author.

\section{REFERENCES}

Abramowitz M., Stegun I. A., 1965, Handbook of Mathematical Functions: with Formulas, Graphs, and Mathematical Tables, revised ed., Dover, New York

Anstey J. et al., 2013, J. Geophys. Res. Atmos., 118, 3956

Armstrong D.J., De Mooij E., Barstow J., Osborn H.P., Blake J., Fereshteh Saniee N., 2017, Nature, 1, 0004.

Asselin R., 1972, Mon. Wea. Rev., 100, 487

Boyd J. P., 2000, Chebyshev and Fourier Spectral Methods, 2nd ed., Dover, New York

Byron, F. W., Fuller, R. W., 1992, Mathematics of Classical and Quantum Physics, Dover, New York

Canuto C., Hussaini M. Y., Qarteroni A., Zang T. A., 1988, Spectral Methods in Fluid Dynamics (New York, NY: Springer)

Cho J. Y-K., Polvani L., 1996, Phys. Fluids, 8, 1531

Cho J. Y-K., Polvani L., 1996, Science, 273, 5273

Cho J. Y-K., Menou K., Hansen B. M. S., Seager S., 2003, ApJ, 587, L117

Cho, J. Y-K. 2008, Phil. Trans. R. Soc. A, 366, 4477

Cho J. Y-K., Menou K., Hansen B. M. S., Seager S., 2008, ApJ, 675,817

Cho J. Y-K., Polichtchouk I., Thrastarson H. Th., 2015, MNRAS, 454,3423

Cho J. Y-K. et al., 2019, Exoplanets and the Sun in Galprin, B. \& Read, P. L. eds., Zonal Jets: Phenomenology, Genesis, and Physics. Cambridge University Press, Cambridge, p. 550

Cho J. Y-K., Skinner J. W., Thrastarson H. Th., 2021, ApJL, 913, L32

Cooper C. S., Showman A. P., 2005, Dynamics and disequilibrium carbon chemistry in hot Jupiter atmospheres, with application to HD 209458b, ApJ, 629, L45-L48

Dang, L., et al., 2018., Nature, 2, 220-227.

Durran D. R., 2010, Numerical Methods for Fluid Dynamics with Applications to Geophysics, 2nd ed., Springer, New York
Eliasen E., Mechenhauer B., Rasmussen E., 1970, Copenhagen Univ., Inst. Teoretisk Meteorologi, Tech. Rep. 2

Gill A.E., 1980, Q. J. Roy. Met. Soc., 106, 447

Grillmair, C. J., et al., 2008., Nature, 456, 767-769.

Holton J. R., 2004, An Introduction to Dynamic Meteorology, 4th ed., Academic Press, San Diego

Heng K., Frierson D., Phillipps P., 2011, MNRAS, 418, 4, 2669-2696

Hogg N. G., Stommel H. M., 1985, Proc. R. Soc. Lond. A, 397, 1

Jackson B., Adams E., Sandidge W., Kreyche S., Briggs J., 2019, ApJ, 157, 239

Jeffreys H., 1925, Proc. R. Soc. London, Ser. A, 107(742), 189-206.

Kizner Z., 2006, Phys. Fluids, 18, 5

Lahaye N., Zeitlin V., 2012, J. Fluid Mech. 706, 71

Liu B., Showman A. P., 2013, ApJ, 770, 42

Matsuno T., 1966, J. Meteorol. Soc. Japan, 44, 25

Menou K., Rauscher E., 2009, ApJ, 700, 887

Mendonça J. M., 2020, MNRAS, 491, 1

Orszag A., 1970, J. Atmos. Sci., 27, 890

Pedlosky J. R., 1987, Geophysical Fluid Dynamics, 2nd ed., Springer-Verlag, New York

Polichtchouk I., Cho J. Y-K., 2012, MNRAS, 424, 2, 1307-1326

Polichtchouk I., Cho Y-K. J., Watkins C., Thrastarson H. Th., Umurhan O. M., Juarez M. T., 2014, Icarus, 229, 355

Rauscher E., Menou K., 2010, ApJ, 714, 1334

Rex D. F., 1950, Tellus, 2:4, 275

Rivier L., Loft R., Polvani L. M., 2002, Mon. Wea. Rev., 130, 1384

Robert A., 1966, J. Met. Soc. Japan, 44, 237

Salby M. L., 1996, Fundamentals of Atmospheric Physics, Academic Press, San Diego

Scott R. K., Rivier L., Loft R., Polvani L. M, 2004, NCAR Technical Note No. 456

Showman A. P., Cooper C. S., Fortney J. J., Marley M. S., 2008, ApJ, 682, 559

Showman A. P., Menou K., Cho, J. Y-K., 2008, in ASP Conf. Ser. 398, Extreme Solar Systems, ed. D. Fischer et al. (San Francisco, CA: ASP)

Showman A. P., Guillot T. 2002, A\&A, 385, 166-180

Showman A. P., Polvani L. M., 2011, ApJ, 738, 71

Skinner J. W., Cho J. Y-K., MNRAS (in press); arXiv:2010.09695

Stern M. E., 1975, J. Mar. Res. 33, 1

Strikwerda J. C., 2004, Finite Difference Schemes and Partial Differential Equations, 2nd ed., Society for Industrial and Applied Mathematics, Philadelphia

Thrastarson H.Th., Cho, J.Y-K. 2010, Apj, 716, 144

Thrastarson H. Th., 2011, PhD. Thesis, Queen Mary University of London

Thrastarson H. Th., Cho J. Y-K., 2011, ApJ, 729, 117

Vallis G. K., 2017, Atmospheric and Oceanic Fluid Dynamics, 2nd ed., Cambridge University Press, Cambridge.

Woolings T., et al., 2018, Cur. Cli. Change Rep., 4, 287

Wu Z., Sarachik E. S., Battisti D.S., 2001, J. Atmos. Sci., 58, 724

Zellem, R. T., et al., 2014. ApJ., 790, 53-62.

Zhang, M., et al., 2018, A.J , 155, 83

\section{APPENDIX}



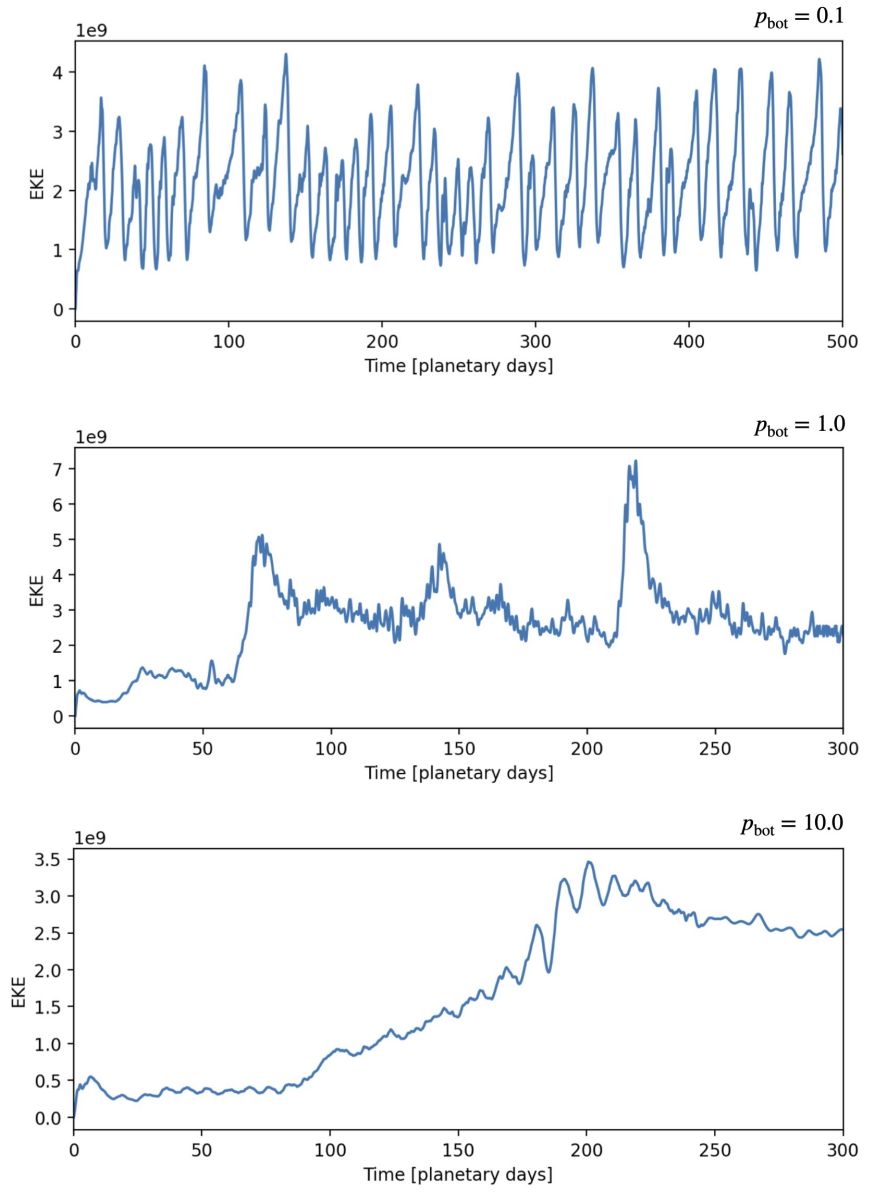

Figure A1. Global eddy kinetic energy time-series for the simulations presented in this paper. The shallow atmosphere (i.e. $p_{\text {bot }}=0.1$ ) simulations equilibrate after $\sim 10$ days. The deep atmosphere simulations (i.e. $\left.p_{\text {bot }}=\{1.0,10.0\}\right)$ show a "jump behaviour" consistent with the temperature time-series (c.f. Fig 13) suggesting multiple "quasi-equilibrium" states over long (Thrastarson 2011) as well as short (Cho, Skinner \& Thrastarson 2021) time-scales. 